Hydroécol. Appl. (1999) Tome 1/2 Vol. 11, pp. 1-28

\title{
Transfert du radiocobalt rejeté par la centrale de Mühleberg dans les systèmes aquatiques de l'Aar et du lac de Bienne (Suisse)
}

\author{
Transport of radiocobalt discharged by the Mühleberg \\ nuclear reactor in the aquatic systems of the Aare river \\ and Lake Biel (Switzerland)
}

\author{
Achim Albrecht(1) \\ (1) Institut fédéral pour l'aménagement, l'épuration et la protection des eaux (EAWAG-ETH) \\ $\mathrm{CH}-8600$ Dübendort, Suisse \\ Adresse actuelle : \\ Institute of Plant Sciences, Swiss Federal Institute of Technology (ETH) \\ Versuchsstation Eschikon, Eschikon 33, Postfach 185 \\ $\mathrm{CH}-8315$ Lindau Switzerland (E-mail : achim.albrecht@ipw.agrl.ethz.ch)
}

Résumé. - Lors d'un rejet d'effluent radioactif, un radioélément donné passe d'un système artificiel, contenu dans la cuve de stockage, à un système naturel. Son comportement n'est donc pas seulement gouverné par des réactions chimiques relativement simples et qui peuvent être reproduites en laboratoire, mais i! est aussi influencé par les mécanismes complexes et encore peu connus qui régissent les systemes naturels. Le cobalt, bien étudié en raison de l'importance radioécologique du ${ }^{60} \mathrm{Co}$ fournit un exemple des réactions chimiques qui jouent un rôle dans un système aquatique, en particulier la complexation organique et inorganique et l'adsorption par la matière en suspension. Sur la base de constantes d'équilibre et d'un simple modèle de complexation de surface, il est possible d'estimer l'importance des espèces chimiques formées par le cobalt.

Les résultats théoriques sont confrontés aux mesures pratiquées in situ en aval de la centrale de Mühleberg, située à proximité de Berne, où l'échantillonnage peut être effectué dans l' Aar et dans le lac de Bienne à $18 \mathrm{~km}$ de la centrale.

Les carottes de sédiments indiquent qu'un faible pourcentage de ${ }^{60} \mathrm{Co}$ se dépose dans certains sites de la rivière (<5\%), alors que la proportion atteint $50 \pm 20 \%$ dans les sédiments du lac de Bienne.

Un modèle de transport est nécessaire pour simuler l'évolution des rejets dans les deux systèmes aquatiques. Le transport en rivière est calculé par les équations d'advection et de dispersion, le transport dans le lac est représenté par le temps de résidence de l'eau et les caractéristiques physiques de ce plan d'eau.

Les mesures ont montré que la fraction du ${ }^{60} \mathrm{Co}$ adsorbée sur les particules $<0.5 \mu \mathrm{m}$ et sur les colloïdes $(0.45 \mu \mathrm{m}$ à 5000 Dalton) ne représente qu'une faible proportion, ne dépassant généralement pas $20 \%$. 
Ces résultats permettent de valoriser les variables les moins bien connues du modèle, comme la concentration de ligands organiques, la constante d'équilibre pour la complexation organique ou la relation entre la masse de particules et le nombre de sites d'adsorption.

Il est important de noter que ce modèle, reposant sur des données collectées in situ, ne s'applique directement qu'à l'Aar en aval de la centrale de Mühleberg et au Lac de Bienne.

Mots-clés. - radiocobalt, système aquatique, complexation-transport, matière en suspension, adsorption-sédimentation.

Abstract. - During the discharge of a radioactive effluent, a radioelement passes from an artificial system in the storage tank, to a natural system. Its behaviour will not only be governed by relatively simple chemical reactions, that can be reproduced in the laboratory, but in addition by more complex, poorly known mechanisms, that control natural systems.

Cobalt, well studied because of the radioecological importance of ${ }^{60} \mathrm{Co}$, represents an example of a metal that participates in chemical reactions which play a role in aquatic systems, particularly organic and inorganic complexation and adsorption on suspended particles. On the basis of equilibrium constants and a simple surface complexation model it is possible to estimate the importance of the different chemical species of cobalt.

The theoretical results are confronted with in situ measurements downstream of the Mühleberg nuclear power plant, situated at close proximity to Berne. Here sampling can be carried out in the river Aare and in Lake Biel, $18 \mathrm{~km}$ downstream of the power plant. Sediment cores indicate that only a small percentage $(<5 \%)$ of ${ }^{\text {fif }} \mathrm{Co}$ is found in riverine sediments, whereas the proportion found in Lake Biel sediments reaches $50 \pm 20 \%$.

A transport model is necessary to simulate the evolution of the discharged water in the two aquatic systems. Transport in the river is calculated on the basis of advection/dispersion equations, transport in the lake is represented by the water residence time and aquatic physical characteristics.

Measurements have shown that the fraction of ${ }^{5(5)} \mathrm{Co}$ adsorbed to particles $<0.5 \mu \mathrm{m}$ and to colloïds $(0.5 \mu \mathrm{m}$ to 5000 Dalton) rarely exceeds $20 \%$.

These results enable evaluating the model variables, which are poorly known, such as the concentration of organic ligands, the equilibrium constant for organic complexation or the relation between particle mass and number of adsorption sites.

It must be remembered that this model, which is based on in situ measurements, can only be directly applied to the Aare downstream of the Mühleberg reactor and Lake Biel.

Key words. - radiocobalt, aquatic system, complexation-transport, suspended particles, adsorption-sedimentation. 


\section{INTRODUCTION}

II est possible de subdiviser tous les sujets d'études des sciences naturelles en deux catégories: les systèmes créés par l'homme et dont il a une connaissance relativement approfondie, et ceux d'origine naturelle. Lors de l'industrialisation des sociétés certaines sciences comme la physique et la chimie ont opté pour l'étude des phénomènes naturels en laboratoire dans un cadre strictement défini, tandis que les géologues et les biologistes ont concentré leurs activités sur la nature, donc sur un cadre mal défini. La simplicité des systèmes fermés des physiciens et chimistes leur a permis de trouver des relations entre les phénomènes, de les décrire mathématiquement et même de faire des pronostics valables, tandis que les géologues et les biologistes avaient du mal à dépasser le niveau de la description. La naissance assez récente de nouvelles voies scientifiques telles que la géochimie ou la chimie aquatique indique que ces deux branches de la science naturelle tentent de se réunir, mais quatre siècles de séparation ne s'effacent pas en deux ou trois décades.

Le comportement du cobalt dans la nature, plus précisément dans le système aquatique, est un sujet idéal pour illustrer ces propos et pour montrer comment un lien entre les scientifiques du laboratoire et de l'ordinateur et leurs collègues du terrain peut aboutir à des résultats enrichissant notre connaissance.

Grâce aux travaux de nombreux géologues, biologistes et géo-biochimistes les concentrations de Co dans les différents roches, sols et plantes sont bien connues (cf. Wedepohl, 1969, 1972, 1974, 1978 1974, 1978; Marschner, 1995; Albrecht, 1999). Cependant, le transfert du cobalt d'un système à l'autre est beaucoup moins bien connu, ce qui tient, sans aucun doute, à la complexité de chaque système, que ce soit l'eau, les sédiments ou le sol. Cette information sur le transfert est essentielle pour les études ecotoxicologiques. Dans la nature, le cobalt stable ne représente aucun danger pour la santé publique, à l'exception de quelques cas rares, les concentrations restent inférieures aux limites des réglementations. La recherche sur le comportement du ${ }^{60} \mathrm{Co}$ dans l'environnement reste indispensable en raison de sa radioactivité $y$ forte et de sa présence dans les installations nucléaires et dans les déchets radioactifs. Dans le milieu aquatique, les interactions du cobalt avec la matière en suspension (MES) et la sédimentation de ces particules jouent un rôle important dans le transfert du métal. La complexation, surtout organique, influence à la fois les processus d'adsorption et d'ingestion par l'organisme. Grâce aux études de laboratoire (Martell et Smith, 1989) et au calcul thermodynamique (Stumm et Morgan, 1996), il est possible de développer des modèles mathémati- 
ques permettant d'évaluer le comportement du cobalt dans un systeme de laboratoire (Müller, 1996; Westall, 1986). Après quelques simplifications, ces programmes servent aussi à faire des prévisions sur le comportement du cobalt dans la nature. Le but de cette publication est de comparer les résultats du terrain avec ceux obtenus par modélisation. L'intérêt porte essentiellement sur les interactions du ${ }^{60} \mathrm{Co}$ avec la matière en suspension et sa forme chimique en solution. Plusieurs méthodes ont été utilisées pour obtenir des résultats in situ sur le comportement $\mathrm{du}{ }^{60} \mathrm{Co}$, rejeté par la centrale: le carottage de sédiments, le traçage et le calcul budgétaire dans les différents compartiments, la sépa- ration de particules et de colloïdes et la spéciation $d u{ }^{60} \mathrm{Co}$ en solution par résine échangeuse d'ions.

\section{MATERIEL, METHODES ET RESULTATS PRECEDENTS}

\subsection{Sédiments}

Les sédiments des rivières et des lacs nous renseignent sur le dépôt des particules et des substances adsorbées en fonction de la profondeur et du temps à condition que les sédiments puissent être datés. A cette fin, plusieurs carottes ont été prélevées dans la retenue hydroélectrique de Niederried et dans le lac de Bienne (Fig. 1). Les carottes de sédiments ont été prises par carottage à

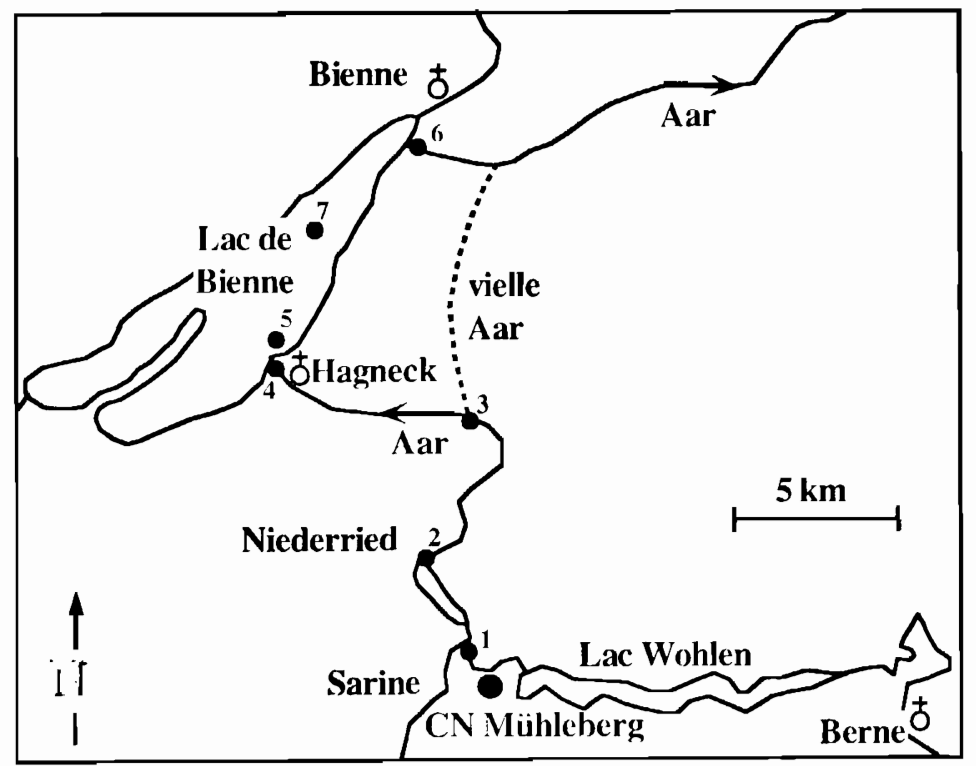

Fig. 1. - Plan de la riviere Aar entre la centrale nucléaire de Mühleberg et le Lac de Bienne avec les lieux d'échantillonnage (ligne en tirets représente le lit naturel de l'Aar).

Fig. 1. - Location map of the river Aare between the nuclear power plant Mühleberg and Lake Biel. including all sampling sites (dashed line represents the natural river bed of the Aare). 
gravité (gravity corer) dans les tubes en polyéthylène avec un diamètre intérieur de $59 \mathrm{~mm}$. Les pertes de sédiments de surface ont été évaluées à l'aide $\mathrm{du}{ }^{7} \mathrm{Be}$, qui se trouve uniquement dans les premiers $\mathrm{cm}$ du sédiment à cause de sa courte période (53 jours). Les carottes ont été sectionnées sur le terrain immédiatement après échantillonnage à l'aide d'une table spécialement conçue. L'épaisseur de chaque tranche variait selon le taux de sédimentation anticipé entre 0.4 et $2 \mathrm{~cm}$. La densité brute a été calculée sur la base du contenu en eau et contrôlée à l'aide de la masse sèche totale et du volume de l'échantillon.

\subsection{Matière en suspension}

Selon Morel et Hering (1993), le mot "particule" signifie une phase ayant une hydrodynamique et thermodynamique différente de celle de la solution. Ces éléments hétérogènes sont habituellement classifiés par leur taille et par leur origine. Dans ce travail les particules et les colloïdes sont traités sans prendre en considération leur origine biologique ou inorganique. La différentiation entre particules et colloïdes est purement arbitraire (opérationnelle).

\subsubsection{Particules}

La matière en suspension est définie de manière arbitraire par sa taille et par l'hydrodynamique du système aquatique. La limite inférieure varie selon les laboratoires et les méthodes utilisées entre 0.2 et $1 \mu \mathrm{m}$ (Hurd et Spencer, 1991). La filtration permet une définition exacte de la limite inférieure, mais elle n'admet pas la récupération d'une masse suffisante pour les différentes analyses en raison du colmatage des filtres. Deux systèmes de centrifugation en continu ont été employés sur le terrain pour séparer la matière en suspension d'un grand volume (plusieurs centaines de litres) d'eau; le système 'LE' de CEPA (Lahn, Forêt Noire) et un système Heraeus" (système H). En utilisant une vitesse de rotation de $30000 \mathrm{rpm}$ (rotations par minute) pour le système LE et $11000 \mathrm{rpm}$ pour le système $\mathrm{H}$ et un débit entre 0.5 et 1 I $\mathrm{min}^{-1}$, la limite de séparation se situe respectivement entre 0.4 à 0.5 $\mu \mathrm{m}$ (Fig. 2) et 0.7 à $1 \mu \mathrm{m}$. Comme la séparation par centrifugation n'est pas seulement basée sur la taille, mais aussi influencée par la densité des particules, qui varie entre 1.05 pour les particules biologiques et 2.7 pour la matière inorganique, ces limites sont transitoires. Une différence significative entre la masse de particules accumulée par les deux systèmes n'a pas été observée, ce qui indique une faible importance de la fraction granulomètrique entre 0.5 et $1 \mu \mathrm{m}$. La limite supérieure des particules qu'on trouve dans les eaux naturelles dépend du courant. Une rivière avec un courant de $1 \mathrm{~m} \mathrm{~s}^{-1}$ à $100 \mathrm{~cm}$ au dessus du lit, arrive à transporter des particules avec un diamètre jusqu'à $3 \mathrm{~mm}$ (Miller et al., 1977). Le volume total de l'eau cen- 


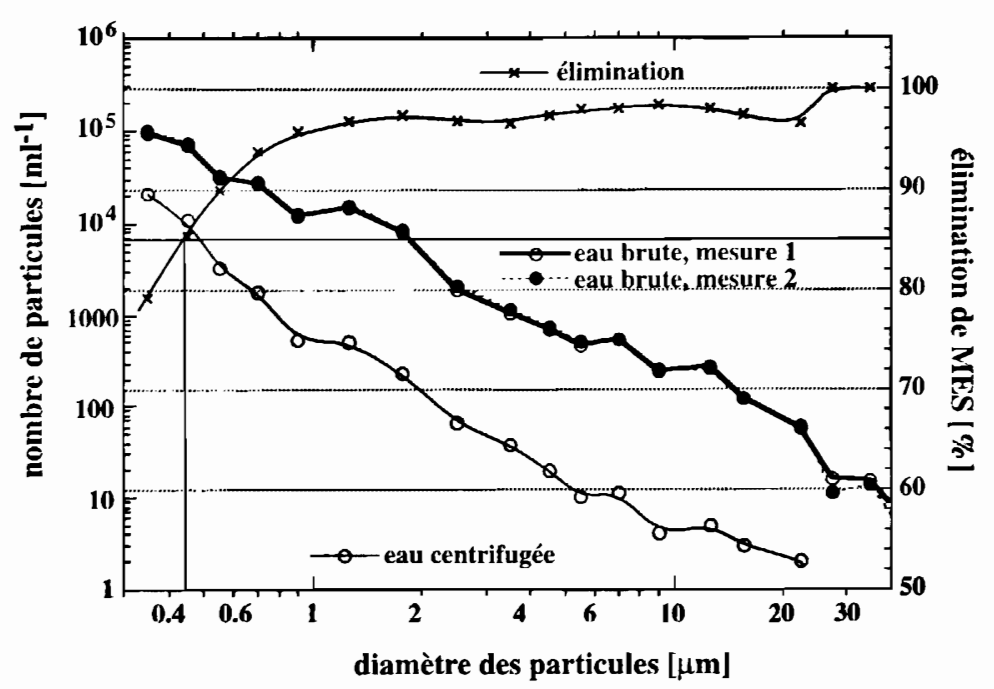

Fig. 2. - Détermination de la limite de séparation de la centrifugeuse en continu (systeme LE) par comparaison du nombre et de la taille des particules dans l'eau brute et centrifugée. L'abscisse montre le diametre des particules, la première ordonnée le nombre de particules par $\mathrm{ml}$ et la deuxième ordonnée l'élimination des particules. En choisissant $85 \%$ comme élimination acceptable, la limite de séparation peut-être définie entre 0.4 et $0.5 \mu \mathrm{m}$ (Albrecht et al., 1996).

Fig. 2. - Determination of the separation limit of the continuous flow centrifuge on the basis of a comparison of size and number of particles in raw and centrifuged water. The $x$ axis indicates the particle diameter, the first $y$ axis the number of particles per $\mathrm{ml}$ and the second $\mathrm{y}$ axis particle elimination. On the basis of $85 \%$, as an acceptable elimination, the separation limit can be defined to be between 0.4 and $0.5 \mu \mathrm{m}$ (Albrecht et al., 1996).

trifugée est mesuré directement. Les particules enrichies par la centrifugation sont recueillies à l'aide d'une feuille de Téflon, le poids est déterminé après lyophilisation.

Pour la filtration in situ on utilise différents filtres de grande taille $(293 \mathrm{~mm}$ de diamètre) qui peuvent être immergés dans l'eau de la rivière ou du lac et employés seul ou en paquet, avec un maximum de 4 filtres par assemblage. Les filtres de $1 \mu \mathrm{m}$ (polyester) et de $0.45 \mu \mathrm{m}$ (Gelman Sciences Supor-450) doivent être rincés, lyophilisés et pesés avant l'utilisation. Le poids des particules est déterminé par la différence de masse après lyophilisation. Les filtres de $1 \mu \mathrm{m}$ peuvent être mesurés directement par spectroscopie $\gamma$ dans une géométrie à puits, les filtres de $0.45 \mu \mathrm{m}$ doivent être calcinés avant la mesure $\%$ Le volume d'eau filtré est mesuré directement sur le terrain.

\subsubsection{Colloïdes}

Les substances qui passent à travers les filtres ou qui ne sont pas centrifugé sont considérées comme 'en solution'. La séparation des colloïdes se fait seulement depuis peu de 
temps par ultrafiltration ou ultracentrifugation avec des artefacts possibles pour chaque méthode (Buesseler et al., 1996; Buffle et al., 1992). La limite inférieure des colloïdes n'est pas clairement définie; une superposition remarquable existe entre le domaine des molécules et des colloïdes (Stumm et Morgan, 1996). Le système employé à l'EAWAG est basé sur la filtration tangentielle (Filtron", Centrasette, 2 membranes de type acrylique avec une surface de $0.46 \mathrm{~m}^{2}$ et une limite de séparation de 5000Dalton). Un flux turbulent d'eau centrifugée passe tangentiellement à la perforation en cycle fermé. Le flux d'eau ultrafiltré se situe entre 0.5 et $1 \mathrm{I} \mathrm{min}^{-1}$. Les substances qui se trouvent dans l'ultrafiltrat, dépourvues de colloïdes, sont également définies comme 'en solution'. La double définition opérationnelle, d'une part pour l'eau filtrée à $0.45 \mu \mathrm{m}$ et d'autre part pour l'eau ultrafiltrée à 5000 Dalton va continuer à exister. La phase colloïdale est désormais concentrée dans le cycle fermé. Le volume restant est autour de 1 litre, avec un volume initial entre 100 et 200 l, le facteur de concentration est dans la plupart des cas supérieur à 100. Pour les analyses des colloïdes, ce volume restant auquel s'ajoutent 2 I d'acide hydrochlorique dilué, utilisé pour le rinçage du systeme, est évaporé. La masse des colloïdes ne peut pas être déterminée de cette manière. La radioactivité associée est donc indiquée en $\mathrm{Bq}$ par volume d'eau traitée. Les pertes durant cette procédure $(5$ à
$10 \%)$ sont calculées par la différence entre l'activité de ${ }^{57} \mathrm{Co}$ et de ${ }^{134} \mathrm{Cs}$ ajoutée dans le concentré colloïdal et mesurée dans le condensé sec.

\subsection{Solution}

Les activités des radionucléides présents dans l'ultrafiltrat sont trop faibles pour une mesure directe par spectroscopie $\gamma$, c'est pourquoi une concentration basée sur la précipitation des hydroxydes de fer est nécessaire. Pour distinguer les différentes formes chimiques, des résines échangeuse d'ion ont été employées.

\subsubsection{Précipitation d'hydroxyde de fer et floculation de montmo- rillonite}

Cette technique s'appuie sur l'efficacité des hydroxydes de fer à adsorber certains métaux, $y$ inclus le cobalt (Dzombak et Morel, 1990). Sur le terrain on ajoute à chaque échantillon d'eau, qu'il soit non traité, centrifugé ou ultrafiltré, un cocktail chimique contenant de l'acide nitrique, du chlorure de fer, d'autres sels de métaux de masse connue $(\mathrm{Be}, \mathrm{Pb}$, Co et $\mathrm{Zn}$ ) et - pour les échantillons traités à partir de 1997 - une activité connue de ${ }^{57} \mathrm{Co}$ et de ${ }^{134} \mathrm{Cs}$. Au laboratoire, l'échantillon est transféré dans un baril ayant un volume de 120 I et mélangé pendant au moins 24 heures. Après cette période d'équilibrage le $\mathrm{pH}$ est porté à 8 en ajoutant de l'ammoniaque. Lors de la précipitation d'hydroxyde de fer, $100 \mathrm{~g}$ d'argile montmorillonite (KSF Fluka") sont 
ajoutés et l'ensemble est agité pendant 24 heures de telle manière que les particules restent en suspension. Le sédiment, qui est composé d'hydroxyde de fer et de montmorillonite, peut être récupéré grâce à un robinet, qui se trouve dans la partie bas du baril en forme d'entonnoir. Ce sédiment est lyophilisé et homogénéisé pour l'analyse spectroscopique $\%$ Le rendement chimique est calculé pour le Co et le Cs en comparaison avec ${ }^{57} \mathrm{Co}$ et ${ }^{134} \mathrm{Cs}$ ajoutés et mesurés et pour $\mathrm{Be}, \mathrm{Pb}, \mathrm{Co}$ et $\mathrm{Zn}$ par l'analyse ICP-AES du sédiment après une nouvelle dissolution en acide chlorhydrique concentré.

\subsubsection{Spéciation de ${ }^{60}$ Co par ré- sine échangeuse d'ion}

Les travaux de Amiard-Triquet et Saas (1979) et de Ferrer (1983) ont clairement démontré que le cobalt peut apparaître sous forme de métal libre et de complexe cationique et anionique. Une étude plus détaillée (Garnier et al., 1997) a pour but de mieux définir les formes complexes en utilisant des résines macroporeuses (cartouches Waters Sep-Pac ${ }^{\mathrm{TM}}$ ) comme le C18, NH2 ou QMA. Ces résines isolent les substances anioniques par interaction électrostatique. QMA est un échangeur anionique à caractère hydrophile, polaire et basique, en mesure de séparer les substances dissoutes anioniques et les acides organiques avec une efficacité de $80 \pm 5 \%$, supérieure à celle des autres résines Waters" (Garnier et al., 1997). Toutes les expériences citées ont été effectuées dans un laboratoire en ajoutant de la radioactivité à un niveau plus élevée que celui qu'on mesure en aval des centrales nucléaires. II a donc été possible de travailler avec des volumes d'eau inférieurs à 1 litre. Pour une analyse in situ en aval de la centrale de Mühleberg, il a fallu de travailler avec des volumes supérieurs à 20 litres. En plus de la résine échangeuse d'ion QMA, des résines BioRad" de type échangeuse d'ion cationique ( $\mathrm{AG} 50 \mathrm{~W}-\mathrm{X} 8, \mathrm{H}^{+}$) et anionique (AG $1 \times 8, \mathrm{Cl}^{-}$en forme neutre) ont été choisies. Le choix de la granulométrie des résines BioRad", 100-200 mesh, est un compromis entre la capacité d'échange (qui augmente avec une granulométrie fine) et l'écoulement (qui décroît avec une granulométrie plus fine). Les résines ont été transférées après rinçage à l'eau distillée dans les tuyaux en PVC de $18.1 \mathrm{~cm}$ de long avec un diamètre intérieur de $2.1 \mathrm{~cm}$, donc un volume humide de résine de $63 \mathrm{~cm}^{3}$. Chaque série est formée par deux tuyaux en série afin de calculer le rendement de séparation. L'eau ultrafiltrée est introduite dans les colonnes de bas en haut à l'aide d'une pompe péristaltique. L'éluat est traité par précipitation d'hydroxyde de fer et floculation de montmorillonite afin de mesurer la radioactivité résiduelle. La radioactivité associée aux résines est déterminée directement par spectroscopie $\gamma$ après homogénéisation. Le rendement (Eff) 
est basé sur l'analyse des résines installées en série en utilisant l'équation suivante :

$$
E f f=1-\frac{B-B l}{A-B l},
$$

où $A$ et $B$ représentent les activitès mesurées des deux résines et $B /$ le blanc de la résine, qui dans tous les cas était inférieur à la limite de détection.

\subsection{Analyses de spectroscopie $\gamma$}

Les activités $y$ ont été mesurées en $\mathrm{Bq} / \mathrm{kg}$ (matière sèche) sur détecteur Ge de haute pureté en géométrie à puits en utilisant les énergies 1173 et $1332 \mathrm{KeV}$ pour le ${ }^{60} \mathrm{Co}$ (période de 5.3 années), 121.8 et $136.2 \mathrm{KeV}$ pour le ${ }^{57} \mathrm{Co}$ (270.9 jours), $661.7 \mathrm{KeV}$ pour ${ }^{137} \mathrm{Cs}$ (30 années), 604.7 et 795.5 $\mathrm{KeV}$ pour le ${ }^{134} \mathrm{Cs}$ (2 années), et 477 $\mathrm{KeV}$ pour le ${ }^{7} \mathrm{Be}$ (53 jours). Les masses variaient entre 0.5 et $10 \mathrm{~g}$. Le Tableau 1 montre le bon accord des résultats de deux intercomparaisons.

\subsection{Modélisation en rivière}

La modélisation en rivière est nécessaire pour évaluer l'heure de l'arrivée et la concentration des radionucléides aux sites d'échantillonnage. La partie modélisée de la rivière Aar s'étend de la Centrale de Mühleberg jusqu'à l'embouchure dans le lac de Bienne (Fig. 1). Le programme d'ordi-

Tableau 1. - Comparaison entre les mesures $\gamma$ de deux intercomparaisons (1, Mundschenk, 1996b; 2 , Ballestra et al., 1997) et les résultats obtenus par l'EAWAG. $A_{k}$ représente la moyenne (en $\mathrm{Bq} \mathrm{kg}^{-1}$ ) de tous les laboratoires participants (à l'exclusion des valeurs douteuses) avec $2 \sigma$ écart-type.

Table 1. - Comparison of $y$ results measured during two intercomparisons (1, Mundschenk (1996b); 2, Ballestra et al. (1997) and those obtained by EAWAG. A $A_{k}$ represents the mean (in $\mathrm{Bq} \mathrm{kg}{ }^{-1}$ ) of all participating laboratories (excluding outlyers) and $2 \sigma$ standard deviation.

$\begin{array}{lrrrrrrrr}\text { radionucleide } & 1 A_{k} & 2 \sigma_{k} & \text { EAWAG: } & 2 \sigma_{k} & 2 A_{k} & 2 \sigma_{k} & \text { EAWAGi } & 2 \sigma_{k} \\ { }^{40} \mathrm{~K} & 683 & 190 & 742 & 41 & 297 & 88 & 350 & 90 \\ { }^{54} \mathrm{Mn} & 4080 & 610 & 4324 & 221 & & & & \\ { }^{57} \mathrm{Co} & 3890 & 810 & 4069 & 212 & & & & \\ { }^{60} \mathrm{Co} & 3380 & 520 & 3571 & 128 & 144 & 40 & 125 & 10 \\ { }^{65} \mathrm{Zn} & 3230 & 530 & 3258 & 168 & & & & \\ { }^{137} \mathrm{Cs} & 43.5 & 7.8 & 45 & 3 & 541 & 214 & 530 & 30 \\ { }^{134} \mathrm{Cs} & & & & & 12 & 4 & 10 & 2 \\ { }^{210} \mathrm{~Pb} & 4510 & 2100 & 4608 & 453 & 30 & 14 & 40 & 20\end{array}$


nateur utilisé, "AQUASIM " et les méthodes de numéri- sation des données géométriques de la rivière sont détaillées par ailleurs (Albrecht et al., 1995; Albrecht, 1998; Reichert, 1994a; Reichert, 1994b). Deux exemples permettent d'illustrer les précisions du modèle (Fig. 3). Dans le premier cas, les rejets de la centrale de Mühleberg ont été modélisés jusqu'à Niederried où un échantillonnage d'eau a permis de comparer les résultats du modèle avec la réalité. De même, le modèle a permis de calculer l'arrivée d'un colorant ajouté dans l'Aar, près de la centrale. Dans les deux cas les courbes de passage (breakthrough curve) modélisées et mesurées sont d'une ressemblance satisfaisante. Les différences sont dues aux phénomènes locaux, qui ne peuvent pas être pris en considération avec le programme unidimensionnel utilisé.

\subsection{Modélisation dans le lac}

Le comportement de l'eau de l'Aar et de la radioactivité transportée est fortement influencé par sa température et par celle du Lac de Bienne et dépend donc principalement de la saison. En été, le lac de Bienne se distingue par une forte stratification. La couche supérieure du lac de Bienne (épilimnion) est en règle générale plus chaude et contient moins de sels dissous que l'eau de l'Aar. L'eau de la rivière s'introduit dans l'épilimnion, au dessus de la thermocline (Fig. 4A). Compte tenu de cette intrusion et du débit relativement élevé de l'Aar par rapport à la taille du lac de Bienne, l'eau de l'affluent ne met guère plus d'une semaine pour transiter durant l'été. Un modèle physique détaillé et les résultats d'un essai de coloration ont confirmé cette hypothèse (Albrecht et al., 1997; Albrecht et al., 1999).

Durant les mois d'hiver, le lac de Bienne n'est que très faiblement stratifié, et la température ne varie que de quelques dixièmes de degrés. Comme la température des deux systèmes aquatiques tombe à environ $4{ }^{\circ} \mathrm{C}$, la salinité devient déterminante pour la densité et, par conséquent, la profondeur de l'intrusion. L'eau de l'Aar, qui contient plus de sels dissous que le lac de Bienne, est donc plus dense et plonge en profondeur durant cette période (Fig. 4B). Le temps de séjour moyen de l'eau de l'Aar dans le lac est de ce fait beaucoup plus long que pendant la période estivale (60 jours).

\section{RESULTATS}

\subsection{Sédiments}

Les résultats détaillés sur la radioactivité des sédiments du réservoir de l'Aar à Niederried et du lac de Bienne ont été publiés antérieurement (Albrecht et al., 1995, 1997, 1998, 1999). Deux exemples sont donnés pour illustrer la datation des sédiments (Fig. 5). Dans les deux cas, l'activité 


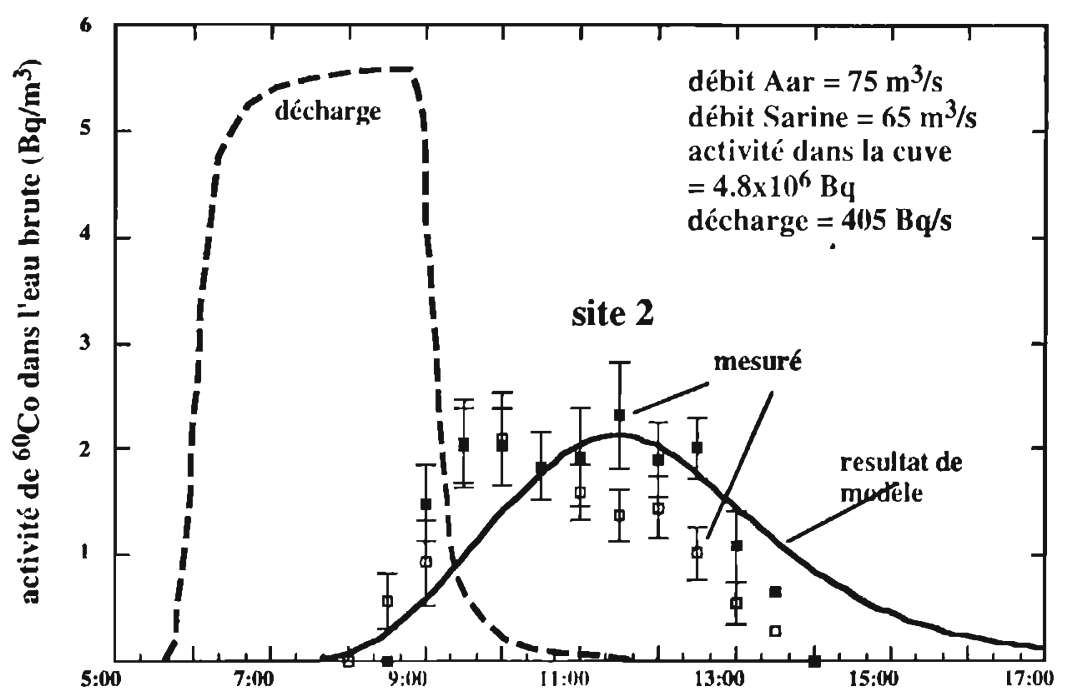

1.4. 94

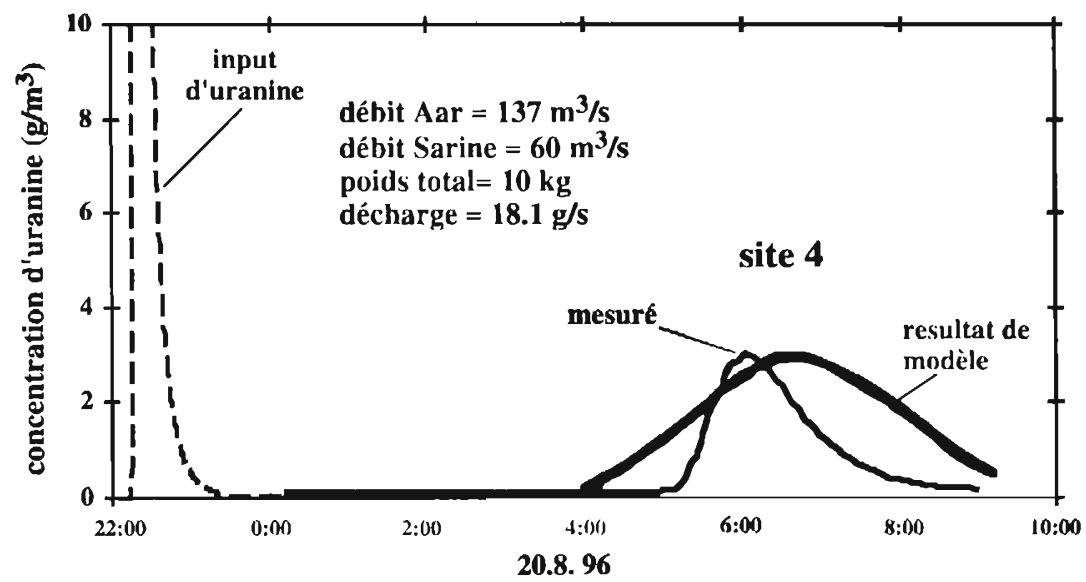

Fig. 3. - Valorisation du modèle de rivière par traçage d'un rejet radioactif au site de Niederried (site 2) et d'une coloration à l'uranine à Hagneck (site 4). Pour chaque expérience, le traceur a été injecté dans l'Aar près de la centrale de Mühleberg. Dans les deux cas, les courbes de passage (breakthrough curve) mesurées el modélisées sont comparables. Les différences sont dues aux phénomènes locaux ou à la désintégration de l'uranine non corrigé.

Fig. 3. - Validation of a river model using a radioactive discharge at the location Niederried (site 2) and uranin at Hagneck (site 4) as tracers. In both experiments the tracer had been added to the Aare river near the Mühleberg power plant. In both cases the measured and modeled breakthrough curves are comparable. Differences are related to local phenomena or the disintegration of uranin. 

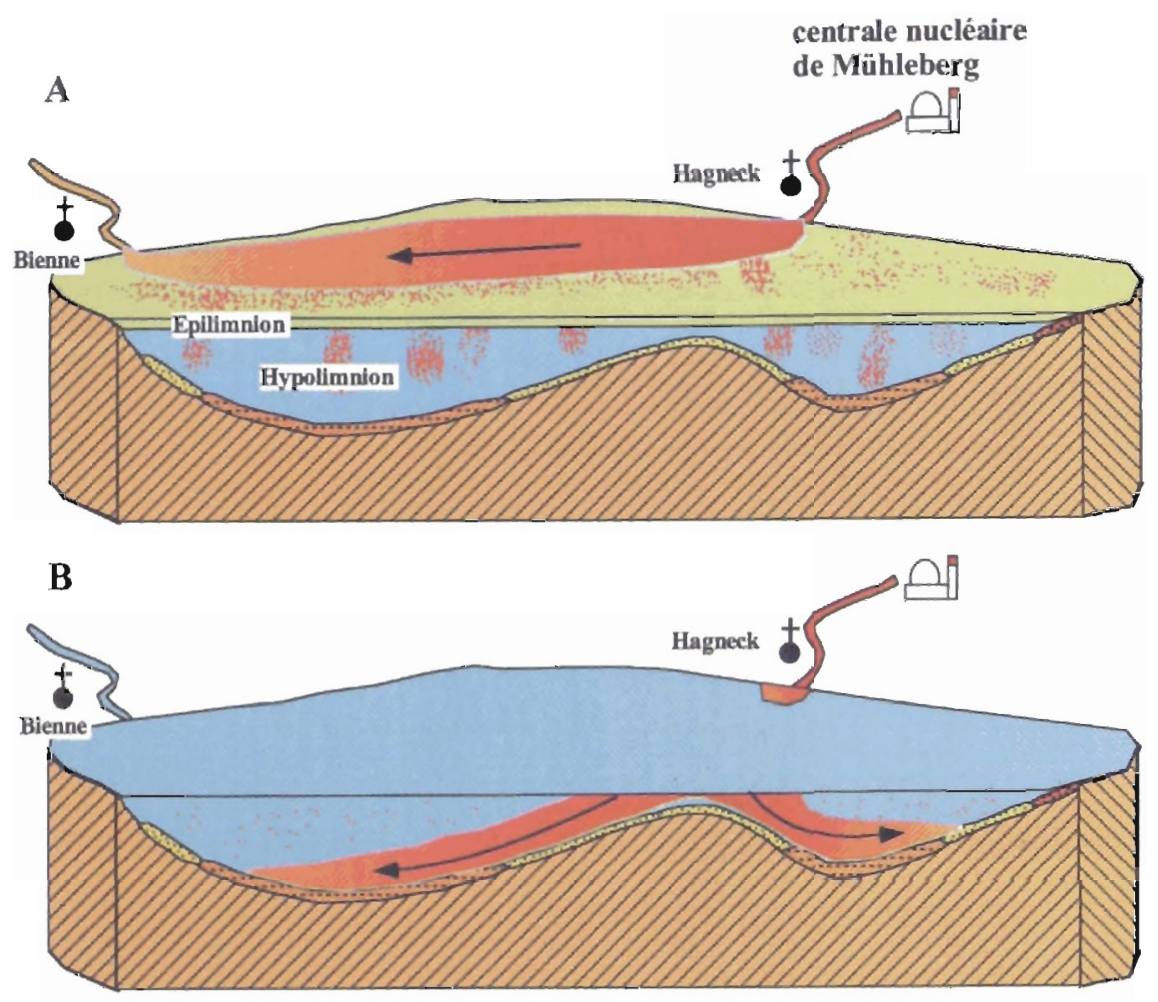

Fig. 4. - Schéma montrant le comportement de l'eau de l'Aar dans le lac de Bienne en période chaude (A) et froide (B).

Fig. 4. - Schematic view of the behaviour of Aare water in lake Biel during the cold (A) and the warm period $(B)$

de ${ }^{60} \mathrm{Co}$ est donnée pour le jour du carottage et pour le sédiment du lac de Bienne, corrigée selon la désintégration radioactive au jour du dépôt. Le flux de ${ }^{60} \mathrm{Co}$ pour une période définie d'une telle carotte se calcule seIon l'équation suivante:

$$
F_{s}=\frac{\sum_{i=1}^{n} \alpha_{i} \rho_{i} \Delta h_{i}}{t_{\text {tol }}},
$$

où $\alpha$ représente l'activité en $B q / \mathrm{kg}$ (corrigée à la date de sédimentation), $p$ la densité sèche du sédiment brut, $\Delta h$ l'épaisseur de chaque échantillon et $t_{\text {tot }}$ le temps représenté par le nombre $n$ d'échantillons de la carotte.

Sur la base de plusieurs carottes représentatives des réservoirs hydroélectriques et du lac de Bienne, un flux moyen est calculé, qui multiplié avec la surface de sédimentation permet l'évaluation de l'inventaire du sys- 
(A) retenu hydrologique activité de ${ }^{60} \mathrm{Co}$ et ${ }^{137} \mathrm{Cs}[\mathrm{Bq} / \mathrm{kg}]$

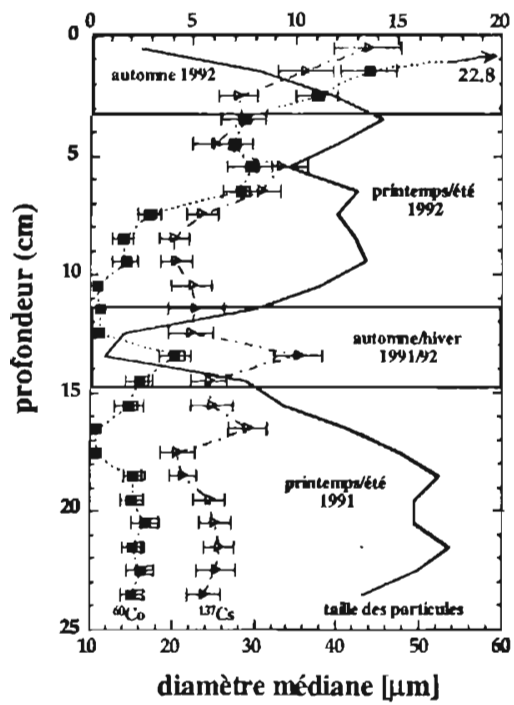

(B) Lac de Bienne

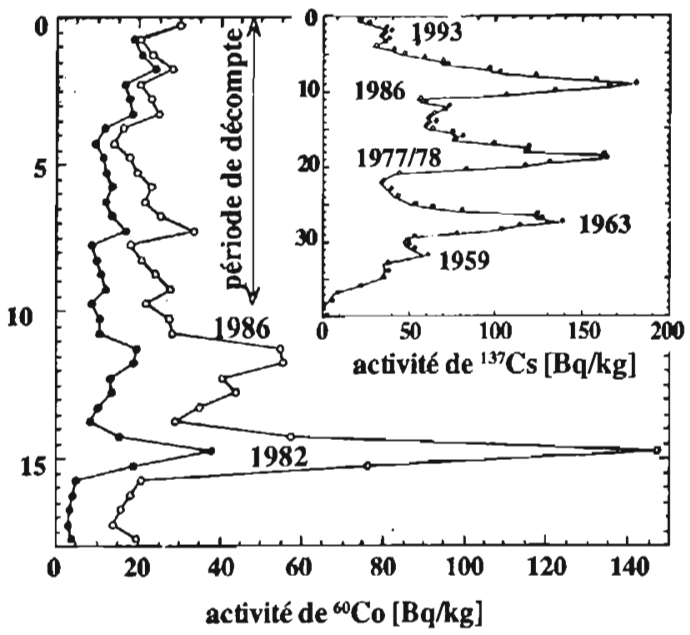

Fig. 5. - Activité de ${ }^{60} \mathrm{Co}$ et de ${ }^{137,134} \mathrm{Cs}$ dans une carotte représentative de la retenue hydroélectrique de Niederried (NR-R2; A) et du lac de Bienne (B). Le sédiment de rivière peut difficilement être daté en utilisant la granulométrie et les variations entre dépôts hivernaux (sédiments argileux et silteux déposés en période de faible débit d'eau) et dépôts estivaux (sédiments silteux et sableux déposés en période de crue). Le sédiment du lac de Bienne est daté à l'aide des maxima de dépôt de radiocaesium liés aux essais nucléaires atmosphériques (maximum en 1963), à l'accident de Tchernobyl (1986) et à une légère augmentation de l'activitè de ${ }^{137} \mathrm{Cs}$ dans les rejets de la centrale de Mühleberg (1977-78). La période de décompte indique la partie de la carotte qui a servi pour le calcul de bilan de ${ }^{60} \mathrm{Co}$.

Fig. 5. - Activity of ${ }^{60} \mathrm{Co}$ and ${ }^{137.134} \mathrm{Cs}$ in a sediment core representing the reservoir of the hydroelectric power plant at Niederried (NR-R2; A) and Lake Biel (B). The riverine sediment can be dated with difficulty using particle grain size and variations between winter (clayey to silty sediments deposited during the period of low run-off) and summer (silty to sandy sediments deposited during high run-off) deposits. The sediment of lake Biel is dated using the maxima of radiocesium deposition related to atmospheric nuclear lesting (maximum 1963), the Chernobyl accident (1986) and a slight increase in the activity of ${ }^{137} \mathrm{Cs}$ rejected by the Mühleberg reactor (1977-78). The 'période de decompte' indicates the core section which served to carry out the inventory calculation for ${ }^{80} \mathrm{Co}$. 
tème en question et une comparaison avec les effluents de la centrale nucléaire. Un tel calcul permet d'évaluer la proportion de ${ }^{60} \mathrm{Co}$ rejetée qui a été déposée avec les sédiments; pour le réservoir de Niederried: $3 \pm 4 \%$ (Albrecht et al., 1995) et pour le lac de Bienne : $40 \pm 15 \%$ (Albrecht et al., 1999).

\subsection{Matière en suspension}

La séparation des particules par centrifugation et par filtration et les mesures d'activité $\gamma$ des deux phases séparées permet d'évaluer les pourcentages de ${ }^{60} \mathrm{Co}$ associé aux particules et à la phase dissoute. Ces résultats (Tableau 2) sont présentés en fonction du temps de contact du ${ }^{60} \mathrm{Co}$ avec l'eau de l'Aar (Fig. 6). Excepté 5 échantillons avec plus de $20 \%$ de ${ }^{60} \mathrm{Co}$ associé aux particules, le taux d'adsorption sur les MES reste inférieur à $20 \%$ sur les autres échantillons qui représentent $92 \%$ de l'effectif total mesuré. Le temps de contact n'influe pas sur le taux d'adsorption ou le coefficient de distribution entre particules et solution $\left(K_{D}\right)$. II est cependant possible d'identifier une dépendance cinétique en comparant la moyenne du taux d'adsorption à un $\mathrm{km}$ de la centrale $(5 \%)$ avec celui des échantillons pris à $18 \mathrm{~km}$ de distance $(15 \%)$. Peu d'échantillons ont indiqué une activité de ${ }^{60} \mathrm{Co}$ supérieure au niveau de détection à cause de la forte dilution de l'eau de l'Aar dans le lac de Bienne (les ré- sultats au niveau de détection ne sont pas indiqué au Tableau 2). Les quelques résultats indiquent, toutefois, que le taux d'adsorption dans le lac reste inférieur à $40 \%$.

La grande variation des $\mathrm{K}_{\mathrm{D}}$ (Fig. 6) ne s'explique ni par la cinétique d'adsorption ni par la concentration en particules qui se situe dans la gamme de 0.3 à $60 \mathrm{mg} \mathrm{l}^{-1}$ (Tableau 2).

Afin d'évaluer l'importance des colloïdes pour le transport du ${ }^{60} \mathrm{Co}, 5$ séparations ont été accomplies sur le trajet de l'Aar entre la centrale et le lac (Tableau 3). Tout comme pour les particules, le taux d'adsorption sur les colloïdes varie fortement, mais n'a pas dépassé $20 \%$ du total de l'activité en ${ }^{60} \mathrm{Co}$ à une exception près. Compte tenu des difficultés d'échantillonnage sur le terrain, les différences entre eau brute et les échantillons d'eau traités sont acceptables.

\subsection{Solution}

Plusieurs tests ont été réalisés avec différentes résines (Tableau 4). Dans une première série de tests, l'eau filtrée à été employée sans séparation de la phase colloïdale. Cette expérience a démontré le changement de la polarité des ions de cobalt ou des complexes auxquels le cobalt est attaché. En utilisant des échangeurs BioRad" cationiques (forme $\mathrm{H}^{+}$) et anionique (forme $\mathrm{Cl}^{-}$). nous avons pu démontrer que le cobalt dans la cuve de stockage était principalement 
Tableau 2. - Résumé des résultats obtenus par centrifugation ( $C F C, H=$ Heraeus, $L=L E$ ) et par filtration (ISF) sur l'adsorption du ${ }^{60} \mathrm{Co}$ sur les matières en suspension en aval de la centrale de Mühleberg $\left(\mathrm{K}_{\mathrm{D}}=\right.$ coefficient de distribution pour ${ }^{60} \mathrm{Co}$ entre matière en suspension et solution).

Table 2. - Summary of results obtained by centrifuging ( $C F C, H=$ Heraeus, $L=L E$ ) and filtration (ISF) on the adsorption of ${ }^{60} \mathrm{Co}$ onto suspended matter downstream of the Mühleberg reactor $\left(K_{D}=\right.$ distribution coefficient for ${ }^{60} \mathrm{Co}$ between suspended particles and solution).

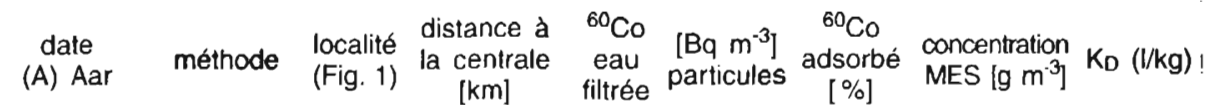

\begin{tabular}{|c|c|c|c|c|c|c|c|c|}
\hline 25.1.1994 & ISF $(1 \mu \mathrm{m})$ & 1 & 0.4 & 235.6 & 1.29 & 0.5 & 0.65 & 8424 \\
\hline 27.10 .1993 & ISF $(1 \mu \mathrm{m})$ & 1 & 0.7 & 3.7 & 0.22 & 5.5 & 1.3 & 45738 \\
\hline 27.10 .1993 & CFC $(\mathrm{H})$ & 1 & 0.7 & 2.94 & 0.29 & 9 & 2.7 & 36533 \\
\hline 25.1.1994 & CFC $(H)$ & 1 & 0.4 & 165.5 & 2.75 & 2 & 1.7 & 9774 \\
\hline 25.1 .1994 & ISF $(1 \mu \mathrm{m})$ & 1 & 1.1 & 516.9 & 4.5 & 1 & 0.58 & 15010 \\
\hline 22.3.1995 & $\operatorname{ISF}(0.45 \mu \mathrm{m})$ & 1 & 0.4 & 2.91 & 0.37 & 11 & 8 & 15012 \\
\hline 22.3.1995 & CFC (L) & 1 & 0.4 & 3.48 & 0.13 & 4 & 4 & 10319 \\
\hline 22.3.1995 & CFC (H) & 1 & 0.4 & 3.32 & 0.14 & 4 & 3 & 14491 \\
\hline 9.9 .1993 & ISF $(1 \mu \mathrm{m})$ & 2 & 4.8 & 39.9 & 4.36 & 10 & 35.9 & 3044 \\
\hline 9.9 .1993 & ISF $(1 \mu \mathrm{m})$ & 2 & 4.8 & 26.1 & 1.84 & 7 & 35.9 & 1964 \\
\hline 1.4.1993 & ISF $(1 \mu \mathrm{m})$ & 2 & 4.8 & 0.6 & 0.06 & 9 & 2.3 & 43478 \\
\hline 1.4.1993 & ISF $(1 \mu \mathrm{m})$ & 2 & 4.8 & 2.1 & 0.1 & 5 & 2.6 & 18315 \\
\hline 1.4 .1993 & ISF $(1 \mu \mathrm{m})$ & 2 & 4.8 & 1.6 & 0.54 & 25 & 2.6 & 129808 \\
\hline 1.4.1993 & ISF $(1 \mu \mathrm{m})$ & 2 & 4.8 & 0.8 & 0.06 & 7 & 2.6 & 28846 \\
\hline 1.4 .1993 & CFC $(H)$ & 2 & 4.8 & 0.83 & 0.21 & 20 & 3.6 & 70281 \\
\hline 9.9 .1993 & $\operatorname{CFC}(H)$ & 2 & 4.8 & 34.86 & 1.54 & 4 & 10.3 & 4289 \\
\hline 17.7 .1996 & CFC (L) & 2 & 4.8 & 2.69 & 0.2 & 6.9 & 2.8 & 26553 \\
\hline 27.10 .1993 & ISF $(1 \mu \mathrm{m})$ & 3 & 10.1 & 3.9 & 0.45 & 10 & 0.6 & 192308 \\
\hline 27.10 .1993 & $\operatorname{CFC}(H)$ & 3 & 10.1 & 10.42 & 0.27 & 3 & 2 & 12956 \\
\hline 9.9 .1993 & CFC $(H)$ & 3 & 10.1 & 16.57 & 15.28 & 48 & 31.3 & 29462 \\
\hline 5.4 .1995 & CFC $(H)$ & 3 & 10.1 & 3.87 & 0.18 & 5 & 10 & 4651 \\
\hline 5.4.1995 & CFC (L) & 3 & 10.1 & 3.41 & 0.27 & 7 & 10 & 7918 \\
\hline 20.7.1995 & CFC (L) & 3 & 10.1 & 2.43 & 0.16 & 6 & 6.05 & 10883 \\
\hline 9.9 .1993 & ISF $(1 \mu \mathrm{m})$ & 4 & 18.3 & 20.3 & 2.73 & 12 & 34.6 & 3887 \\
\hline 9.9 .1993 & ISF $(1 \mu \mathrm{m})$ & 4 & 18.3 & 20.4 & 3.22 & 14 & 54.7 & 2886 \\
\hline 27.10 .1993 & ISF $(1 \mu \mathrm{m})$ & 4 & 18.3 & 5.5 & 0.36 & 6 & 5.1 & 12834 \\
\hline 27.10 .1993 & ISF $(1 \mu \mathrm{m})$ & 4 & 18.3 & 6.3 & 0.11 & 2 & 3.1 & 5632 \\
\hline 1.4.1993 & $\operatorname{CFC}(\mathrm{H})$ & 4 & 18.3 & 1.17 & 0.18 & 13 & 3.2 & 48077 \\
\hline 1.4.1993 & $\operatorname{CFC}(H)$ & 4 & 18.3 & 0.59 & 0.1 & 14 & 2.7 & 62775 \\
\hline
\end{tabular}




\begin{tabular}{|c|c|c|c|c|c|c|c|c|}
\hline $\begin{array}{l}\text { date } \\
\text { (A) Aar }\end{array}$ & méthode & $\begin{array}{l}\text { localité } \\
\text { (Fig. 1) }\end{array}$ & $\begin{array}{c}\text { distance à } \\
\text { la centrale } \\
{[\mathrm{km}]}\end{array}$ & $\begin{array}{l}{ }^{60} \mathrm{Co} \\
\text { eau } \\
\text { filtrée }\end{array}$ & $\begin{array}{l}{\left[\mathrm{Bq} \mathrm{m} \mathrm{m}^{-3}\right]} \\
\text { particules }\end{array}$ & $\begin{array}{c}{ }^{60} \mathrm{Co} \\
\text { adsorbé } \\
{[\%]}\end{array}$ & $\begin{array}{l}\text { concentration } \\
\text { MES }\left[\mathrm{g} \mathrm{m}^{-3}\right]\end{array}$ & $K_{D}(1 / k g) !$ \\
\hline 9.9 .1993 & CFC (H) & 4 & 18.3 & 9.2 & 2.02 & 18 & 16.6 & 13227 \\
\hline .9 .1993 & CFC $(H)$ & 4 & 18.3 & 14.57 & 2.19 & 13 & 29.5 & 5095 \\
\hline 27.10 .1993 & $\operatorname{CFC}(H)$ & 4 & 18.3 & 5.55 & 0.3 & 5 & 2.6 & 20790 \\
\hline 20.8 .1996 & CFC (L) & 4 & 18.3 & 0.47 & 0.65 & 58 & 6.6 & 209542 \\
\hline \multicolumn{9}{|l|}{$\begin{array}{l}\text { (B) lac de } \\
\text { Bienne }\end{array}$} \\
\hline 10.9.1993 & ISF $(1 \mu \mathrm{m})$ & 5 & 19.0 & 0.34 & 0.11 & 25 & 2.51 & 134139 \\
\hline .9 .1993 & ISF $(1 \mu \mathrm{m})$ & 5 & 19.0 & 1.08 & 0.63 & 37 & 16.59 & 35193 \\
\hline 9.1993 & CFC $(H)$ & 6 & 27.0 & 3.81 & 0.13 & 3 & 4.192 & 8325 \\
\hline 8.1994 & CFC (L) & 7 & 23.0 & 0.15 & 0.03 & 17 & 0.35 & 571429 \\
\hline
\end{tabular}

Tableau 3. - Résultats des séparations combinées de particules et de colloïdes.

Table 3. - Results of combined separations of particles and colloids

\begin{tabular}{|c|c|c|c|c|c|c|c|c|c|c|}
\hline \multirow[t]{2}{*}{ date } & \multirow[t]{2}{*}{$\begin{array}{l}\text { localité } \\
\text { (Fig. 1) }\end{array}$} & \multirow{2}{*}{$\begin{array}{c}\text { temps } \\
\text { de } \\
\text { contact } \\
{[\mathrm{h}]}\end{array}$} & \multicolumn{2}{|c|}{ particules } & \multicolumn{2}{|c|}{ colloïdes } & \multicolumn{2}{|c|}{ en solution } & \multirow{2}{*}{$\begin{array}{c}\text { eau } \\
\text { brute } \\
{\left[\mathrm{Bq} \mathrm{\textrm {m } ^ { - 3 }}\right]}\end{array}$} & \multirow{2}{*}{$\begin{array}{c}\text { différence } \\
\text { au brute } \\
\text { et eau } \\
\text { traitée } \\
{[\%]}\end{array}$} \\
\hline & & & {$\left[\mathrm{Bq} \mathrm{m} \mathrm{m}^{-3}\right]$} & [\%] & {$\left[\mathrm{Bq} \mathrm{\textrm {m } ^ { - 3 }}\right]$} & [\%] & {$\left[\mathrm{Bq} \mathrm{m} \mathrm{m}^{-3}\right]$} & [\%] & & \\
\hline 3.1995 & 1 & 0.3 & 0.13 & 3.6 & 0.15 & 4.2 & 2.71 & 75.1 & 2.99 & 17 \\
\hline 4.1995 & 3 & 4.8 & 0.27 & 4.5 & 0.04 & 0.7 & 3.4 & 56.1 & 3.71 & 39 \\
\hline .7 .1995 & 3 & 4.5 & 0.16 & 6.1 & 0 & 0.0 & 2.43 & 92.7 & 2.59 & 1 \\
\hline 7.7996 & 2 & 2 & 0.2 & 6.9 & 0.4 & 13.8 & 2.3 & 79.3 & 2.9 & - \\
\hline 2.7 .1997 & laboratoire & 24 & 6682 & 29.5 & 508 & 2.2 & 15435 & 68.2 & 22625 & - \\
\hline 10.1997 & 4 & 16 & 1.66 & 15.3 & 3.5 & 32.3 & 5.69 & 52.4 & 10.85 & -65 \\
\hline
\end{tabular}

cationique, et qu'un changement considérable se produit après contact avec l'eau de l'Aar. Dans un premier test $51 \%$ du ${ }^{60} \mathrm{Co}$ total 'en solution' était sous forme anionique, dans un deuxième test $77 \%$; ces résultats sont semi-quantitatifs en raison de la présence de la phase colloïdale et de l'adsorption de ${ }^{60} \mathrm{Co}$ aux parois des bouteilles lors du transport au labo- ratoire et au stockage (Albrecht et al., 1994 ; Fischer et Albrecht, résultats non publiés). Ces résultats ont démontré que les séparations doivent être accomplies directement sur le terrain ou immédiatement après le transport au laboratoire, ce qui a été fait pour les tests suivants pour lesquels la phase colloïdale était également séparée. 


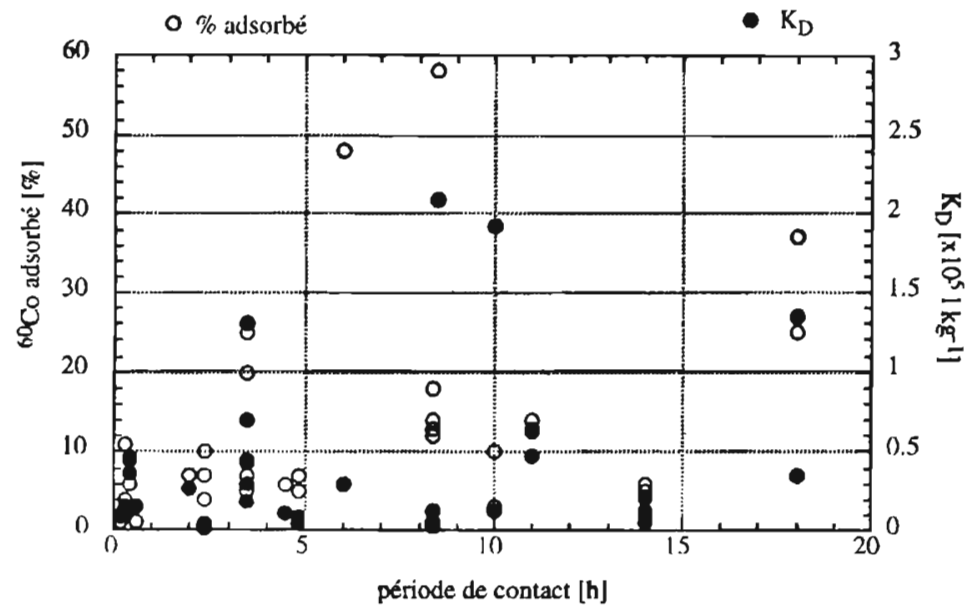

Fig. 6. - Le comportement du ${ }^{60} \mathrm{Co}$ dans l'eau de l'Aar en aval de la centrale de Mühleberg est représenté par le pourcentage de ${ }^{60} \mathrm{Co}$ adsorbé sur les matieres en suspension (> 0.5 , respectivement $1.0 \mu \mathrm{m}$ de diamètre selon type de filtre utilisé) et par le $\mathrm{K}_{\mathrm{D}}$, en fonction du temps de contact avec l'eau de la rivière.

Fig. 6. - Behavior of ${ }^{60} \mathrm{Co}$ in Aare water downstream of the Mühleberg nuclear reactor, percentage of ${ }^{60} \mathrm{Co}$ adsorbed to suspended matter (>0.5, respectively $1.0 \mu \mathrm{m}$ in diameter depending on filter type used) and $K_{D}$ as a function of contact time with river water.

Le Tableau 4 indique les pertes de ${ }^{60}$ Co lors du stockage de l'eau filtrée, surtout les 3 premiers tests lorsque l'échantillon a été entreposé pendant plusieurs jours, voir semaines, montrent que les pertes peuvent atteindre $90 \%$. La réduction de ces pertes permet une interprétation plus quantitative. La moyenne de tous les tests indique que $60 \%$ du ${ }^{60} \mathrm{Co}$ est sous forme complexée et $40 \%$ sous forme libre. Les résines spécifiques employées par Garnier et al. (1997) ne peuvent cependant pas être utilisées sur le terrain à cause de leur granulométrie fine, qui ne permet pas un écoulement convenable pour les volumes requis par les faibles activités en nature. Pour le test du 22.7.95, une activité élevée a été employée permettant une mesure d'activité des résines après un traitement de $20 \mathrm{I}$. Pour les séparations sur le terrain avec les échantillons de faible activité, des volumes supérieurs à 60 I sont recommandés.

\subsection{Modélisation du Co dans un système 'simple'}

Le cobalt existe, dans un système 'simple', sous forme d'ions ou de complexes inorganiques. Les réactions chimiques au laboratoire sont connues et les constantes thermodynamiques ont été déterminées (i.e. Martell et Smith, 1989; Morel et Hering, 1993; Stumm et Morgan, 1996). A partir de l'équilibre thermodynami- 
Tableau 4. - Spéciation du ${ }^{60}$ Co dans la phase dissoute par échange sur des résines organiques; " en laboratoire; "* calculée par différence, résine anionique = BioRad" $\mathrm{Cl}^{-}$form, sauf 22.7.97, $\mathrm{QMA}$; résine cationique = BioRad" $\mathrm{H}^{+}$form).

Table 4. - Speciation of ${ }^{60} \mathrm{Co}$ in the dissolved phase obtained by exchange with organic resins; * in the laboratory ; ${ }^{\star}$ calculated by difference, anionic exchange resin = BioRad" $\mathrm{Cl}^{-}$form, except 22.7.97, QMA; cationic exchange resin = BioRad" $\mathrm{H}^{+}$form).

$\begin{array}{ccccccccc}\begin{array}{c}\text { date } \\ \text { d'échan- } \\ \text { tillonage }\end{array} & \begin{array}{c}\text { date de } \\ \text { sépa- } \\ \text { ration } \\ \text { chimique }\end{array} & \begin{array}{c}\text { localité } \\ \text { (Fig. 1) }\end{array} & \begin{array}{c}\text { eau } \\ \text { filtrée/ } \\ \text { ultrafiltrée } \\ \mathrm{Bq} / \mathrm{m}^{3}\end{array} & \begin{array}{c}\mathrm{Bq} / \mathrm{m}^{3} \\ \text { anionique/complexé }\end{array} & \begin{array}{c}\% \\ \text { cationique/libre }\end{array} & \begin{array}{c}\text { différence: } \\ \text { eau brute } \\ \text { et eau }\end{array} \\ 25.1 .94 & 28.1 .94 & \text { 1* }^{*} & 230.9 & 91.9 & 51 & 88.8 & 49 & 22 \\ 25.1 .94 & 2.2 .94 & 1^{*} & 228.6 & 134.4 & 77 & 40.8 & 23 & 23 \\ 25.1 .94 & 19.2 .94 & 1^{*} & 232.2 & 9.9 & 41 & 14.0 & 59 & 90 \\ 22.7 .97 & 23.7 .97 & - & 15435 & 5351 & 35 & 10084 & 65 & 0^{* *} \\ 20.7 .95 & 20.7 .95 & 3 & 2.46 & 1.49 & 61 & 0.94 & 39 & 1 \\ 8.10 .97 & 8.10 .97 & 4 & 5.69 & 4.6 & 81 & 1.09 & 19 & 0^{* *}\end{array}$

que, il est possible de décrire et de modéliser ces réactions et de prévoir l'importance relative des différentes formes chimiques (Westall et al., 1976; Westall, 1986). L'exemple suivant d'une complexation inorganique permet d'expliquer l'approche :

$\mathrm{Co}^{2+}+\mathrm{H}_{2} \mathrm{O} \Leftrightarrow \mathrm{CoOH}^{+}+\mathrm{H}^{+}$

$\log K=-9.7$

Cette réaction dépend $\mathrm{du} \mathrm{pH}$ et le complexe inorganique $\mathrm{CoOH}^{+}$est plus stable dans un milieu basique. Les calculs présentés ici ont été faits avec le programme ChemEQL (Müller, 1996). Bien que Co(III) soit présent dans certaines cuves de stockage des réacteurs nucléaires et dans les eaux en aval de ces réacteurs (Leonard et al., 1993a; Leonard et al., 1993b), sa rareté permet d'exclure la forme oxydée dans les calculs de modèle, donc de négliger les réactions d'oxydoréductions. II est difficile de modéliser ces réactions, car elles sont fortement influencées par des processus biochimiques, qui ne suivent pas les lois de la thermodynamique (Moffett et Ho, 1996). Outre les réactions entre différentes formes inorganiques, le programme permet également de modéliser l'adsorption sur les particules et la complexation organique. Les réactions chimiques suivantes illustrent la méthode:

$$
\begin{array}{r}
\equiv \mathrm{FeOH}+\mathrm{Co}^{2+} \Leftrightarrow \mathrm{FeOCo}^{+}+\mathrm{H}^{+} \\
\log \mathrm{K}=-0.46 \\
\mathrm{~L}+\mathrm{Co}^{2+} \Leftrightarrow \mathrm{CoL} \quad \log \mathrm{K}=10.55
\end{array}
$$

Adsorption et complexation organique sont représentées par des équations chimiques avec leurs constantes d'équilibre. $\equiv \mathrm{FeOH}$ représente un groupe $\mathrm{OH}$ de surface, qui peut réagir comme acide ou comme base, $L$ représente tous les ligands organiques possibles, permettant la complexation 
des métaux. Comme les propriétés chimiques de surface des oxydes sont particulièrement bien étudiées (Dzombak et Morel, 1990), elles sont souvent utilisées comme modèle. La constante d'équilibre (Log $K=0.46$ ) s'applique bien sûr uniquement aux oxydes de fer utilisés et aux conditions chimiques de l'expérience, mais la complexité de la composition chi- mique et physique des assemblages naturels de particules et la lenteur des réactions ( $\mathrm{Li}$ et al., 1984; Mundschenk, 1996a) rendent la détermination des constantes d'équilibre très difficile. La concentration des sites d'adsorption dépend naturellement du type de l'échantillon, l'utilisation des constantes obtenues pour les oxydes de fer $\left(2 \times 10^{-4} \mathrm{~mol} / \mathrm{g}\right)$

Tableau 5. - Tableau des équilibres indiquant les composants et leurs concentrations molaires, les espèces, leurs réactions et les log $\mathrm{K}$ correspondants (voir Sigg et al., 1994 pour plus de détail ; $S \& M=S m i t h$ et Martell, 1974; D\&M=Dzombak et Morel, 1990; $\mathrm{Q}=$ Qian et al., 1998).

Table 5. - Equilibrium Tableau, indicating the components and their molar concentrations, the species, their reactions their corresponding log K (voir Sigg ef al., 1994 pour plus de détail ; $S \& M=S m i t h$ et Martell, 1974 ; $D \& M=D z o m b a k$ et Morel, 1990; Q=Qian et al., 1998).

\begin{tabular}{|c|c|c|c|c|c|c|c|}
\hline & $\begin{array}{l}\mathrm{Co}^{2+} \\
\text { total }\end{array}$ & $\begin{array}{l}\mathrm{H}_{2} \mathrm{CO}_{3} \\
\text { total }\end{array}$ & $\begin{array}{l}=\mathrm{FeOH} \\
\text { total }\end{array}$ & $L$ total & $\mathrm{H}^{+}$total & $\log K$ & référence \\
\hline $\mathrm{Co}^{2+}$ & 1 & 0 & 0 & 0 & 0 & 0 & \\
\hline $\mathrm{CoOH}^{+}$ & 1 & 0 & 0 & 0 & -1 & -9.7 & S\&M \\
\hline $\mathrm{Co}(\mathrm{OH})_{2}(\mathrm{aq})$ & 1 & 0 & 0 & 0 & -2 & -19.6 & S\&M \\
\hline $\mathrm{Co}(\mathrm{OH})_{3}{ }^{-}$ & 1 & 0 & 0 & 0 & -3 & -32.3 & S\&M \\
\hline $\mathrm{Co}(\mathrm{OH})_{4}{ }^{2-}$ & 1 & 0 & 0 & 0 & -4 & -45.8 & S\&M \\
\hline $\mathrm{Co}_{2} \mathrm{OH}_{3}{ }^{+}$ & 2 & 0 & 0 & 0 & -1 & -11.3 & S\&M \\
\hline $\mathrm{CO}_{4}(\mathrm{OH})_{4}^{4+}$ & 4 & 0 & 0 & 0 & -4 & -30.4 & S\&M \\
\hline $\mathrm{H}_{2} \mathrm{CO}_{3}$ & 0 & 1 & 0 & 0 & 0 & 0 & \\
\hline $\mathrm{HCO}_{3}{ }^{\circ}$ & 0 & 1 & 0 & 0 & -1 & -6.35 & S\&M \\
\hline $\mathrm{CO}_{3}{ }^{2-}$ & 0 & 1 & 0 & 0 & -2 & -16.68 & S\&M \\
\hline $\mathrm{OH}-$ & 0 & 0 & 0 & 0 & -1 & -14 & \\
\hline$=\mathrm{FeOH}$ & 0 & 0 & 1 & 0 & 0 & 0 & \\
\hline$=\mathrm{FeOCo}^{+}$ & 1 & 0 & 1 & 0 & -1 & -0.46 & $D \& M$ \\
\hline L & 0 & 0 & 0 & 1 & 0 & 0 & \\
\hline CoL & 1 & 0 & 0 & 1 & 0 & 9.6 & Q \\
\hline \multirow[t]{2}{*}{$\mathrm{H}^{+}$} & 0 & 0 & 0 & 0 & 1 & 0 & \\
\hline & $2 \times 10^{-9}$ & $1 \times 10^{-5}$ & $4 \times 10^{-7}$ & $7 \times 10^{-10}$ & & & \\
\hline
\end{tabular}


représente donc une nouvelle simplification.

De même, la complexation organique a été simplifiée radicalement dans l'équation. Le groupement de toutes les substances organiques dissoutes dans un terme (L) n'est pas justifié en raison de la complexité de ces substances (Buffle, 1988; Tipping et Hurley, 1992). La constante d'équilibre 'conditionnelle' pour la complexation organique a été évaluée pour le $\mathrm{Co}_{\text {stable }}$ dans le système aquatique suisse sur la base de mesures de voltamétrie $(\log \mathrm{K}=9.5-11.6$ pour $\mathrm{pH} 8)$; ainsi que la concentration de ligands $([L]=1.4-7.6 \mathrm{nM})$; Qian et al., 1997). La concentration de $\mathrm{Co}_{\text {total }}$ est égale à la concentration de $\mathrm{Co}_{\text {stable }}$ qui varie entre 1 et $3.3 \mathrm{nM}$ dans le système étudié (Qian et al., 1998). Une comparaison avec des mesures in situ permettra une évaluation de cette simplification. L'ensemble des espèces chimiques, les concentrations, les réactions et les constantes d'équilibres se trouvent dans le Tableau 5 .

Les résultats donnés par le modèle pour un systeme sans adsorption ni complexation organique (Fig. 7A) indiquent clairement la prédominance de Cobalt libre $\left(\mathrm{Co}^{2+}\right)$ ou sa forme hydratée au pH du système naturel. Des produits de l'hydrolyse, comme $\mathrm{CoOH}^{+}$apparaissent uniquement aux $\mathrm{pH}$ élevés. La présence de particules (Fig. $7 \mathrm{~B}$ ) réduit la proportion de $\mathrm{Co}^{2+}$ surtout pour l'intervalle du $\mathrm{pH}$ qu'on trouve dans l'Aar. A pH 8, plus de $90 \%$ du Co ont, selon le calcul, une préférence pour la phase solide. La présence de ligands organiques change brusquement la spéciation du cobalt (Fig. 7C). Le calcul, qui est basé sur les données du Tableau 5 (à $\mathrm{pH}$ 8), indique que $80-90 \%$ du Co total est de préférence complexé par des ligands organiques, $10 \%$ adsorbé aux particules et $1-2 \%$ libre.

\section{DISCUSSION ET CONCLUSIONS}

\subsection{Comparaison entre modèle et résultats de terrain}

Une comparaison entre les conclusions du modèle et les résultats du terrain permet de tester la validité du modèle. Dans le système où le cobalt, sous forme libre ou de complexe inorganique (Fig. $7 \mathrm{~B}$ ), peut réagir uniquement avec des surfaces de matière en suspension, le modèle indique que plus de $90 \%$ sont adsorbés. Ceci est clairement en contradiction avec les résultats d'analyse de particules et de colloïdes, qui ont affiché une moyenne de ${ }^{60} \mathrm{Co}$ adsorbé de $15 \%$ pour les particules et de $20 \%$ pour la somme de particules et de colloïdes. II est possible qu'on ait réalisé toutes ces séparations à courte distance de la centrale nucléaire de Mühleberg et que l'équilibre, qui est à la base du modèle, n'était pas encore atteint. C'est grâce au bilan du ${ }^{60} \mathrm{Co}$ dans les sédiments du lac de Bienne, indiquant que $50 \%$ du ${ }^{60} \mathrm{Co}$ rejeté ne participent pas aux réactions d'adsorption et de sédimentation, malgré leur longue résidence, 


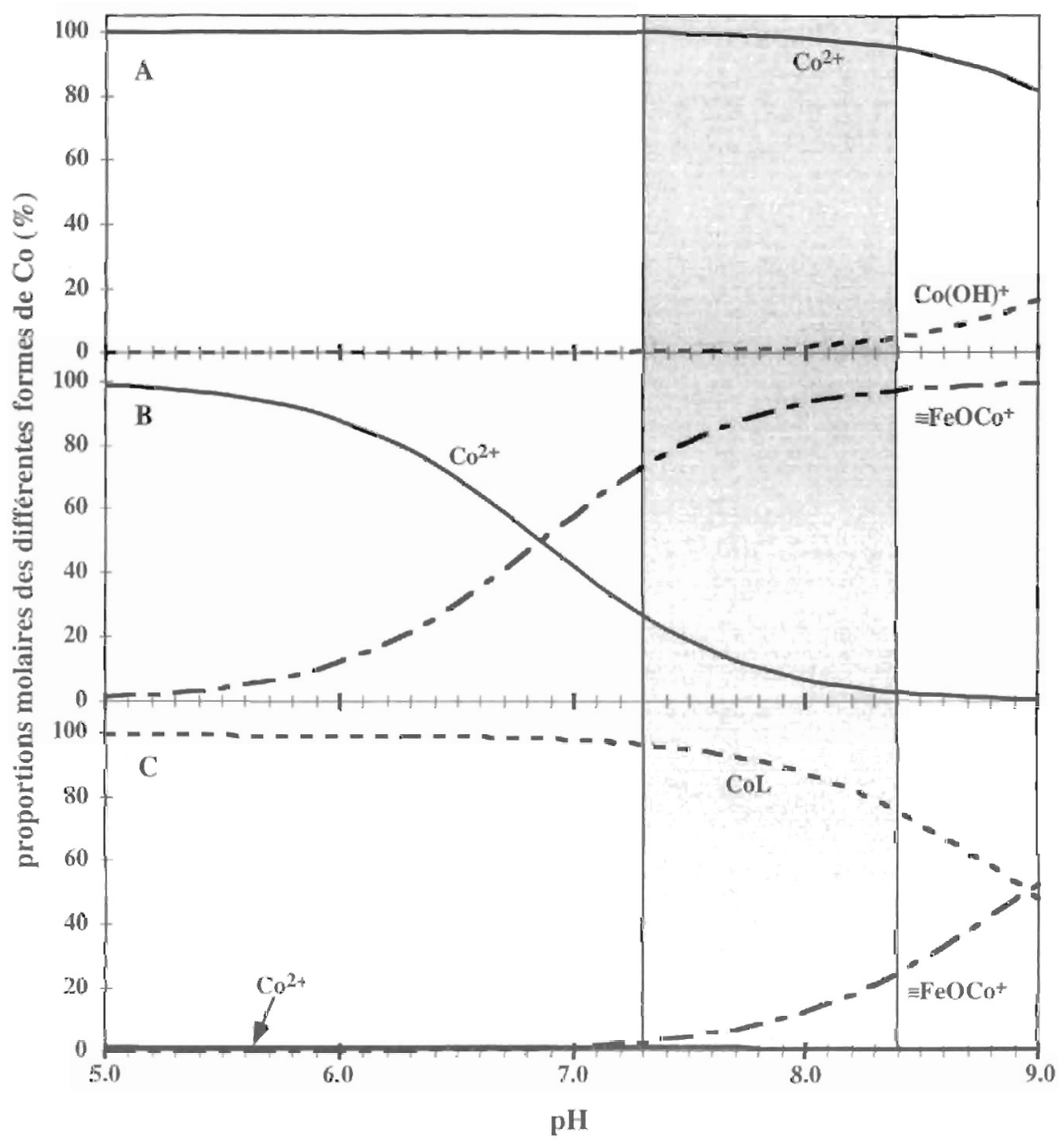

Fig. 7. - Modèle du comportement du cobalt (2nM) dans un système simple (A) dominé par $\mathrm{H}_{2} \mathrm{O}$ et $\mathrm{H}_{2} \mathrm{CO}_{3}, 1 \times 10^{5} \mathrm{M}$; (B) en présence de particules (oxydes de fer; concentration de particules = $2 \mathrm{mg} \mathrm{l}^{-1}$, densité de sites d'adsorption $=2 \times 10^{-4} \mathrm{~mol} \mathrm{~g}^{-1}$ ) et (c) de ligands organiques (concentration de ligands $=4.5 \mathrm{nM}$ ).

Fig. 7. - Model of the behaviour of cobalt $(2 n M)$ in a simple system (A) dominated by $\mathrm{H}_{2} \mathrm{O}$ and $\mathrm{H}_{2} \mathrm{CO}_{3}, 1 \times 10^{5} \mathrm{M}$; (B) in the presence of particles (iron oxide; particle concentration $=2 \mathrm{mg} \mathrm{I}^{-1}$, adsorption site density $=2 \times 10^{-4} \mathrm{~mol} \mathrm{~g}^{-1}$ ) and $(\mathrm{c}$ ) organic ligands (ligand concentration $=4.5 \mathrm{nM}$ ).

que ce modèle le plus simpliste peutêtre rejeté.

C'est donc, la compétition entre les phénomènes d'adsorption et de complexation organique qui gouverne le devenir du radiocobalt. L'exemple représenté dans la Fig. $7 \mathrm{C}$ indique pour un $\mathrm{pH} 8$ un taux d'adsorption de $15 \%$, comparable avec la moyenne de $20 \%$ des mesures in situ, mais 
avec moins de $2 \% \mathrm{Co}^{2+}$, sensiblement inférieure aux $30 \%$ observés sur le terrain. Parmi les variables qui agissent sur le système complexe du Co (coefficient $\mathrm{K}$ des réactions d'adsorption et de complexation, concentration des sites d'adsorption et de ligands, Tableau 5), c'est la concentration en ligands qui influence le plus le système. Si l'on prend le minimum et le maximum de la concentration des ligands organiques publiés par Qian et al. (1998, 1.4 et $7.6 \mathrm{nM}$ ), la différence des résultats du modèle est considérable (Fig. 8). La proportion de Co associé aux ligands organiques varie entre 49 et $93 \%$, et la proportion de Co associé aux particules entre 6 et $47 \%$ (Tableau 6 ). Cette variation reflète les valeurs trouvées dans l'Aar en aval de la centrale de Mühleberg pour l'adsorption et la complexation organique. Les valeurs de terrain pour le Co libre sont plus élevées (30\%) que les valeurs modélisées (maximum $3 \%$ ). Cette différence peut très bien être expliquée par le déséquilibre chimique en aval de la centrale et la prédominance de Co libre dans la cuve de stockage.
$\mathrm{Vu}$ l'importance des ligands, une analyse de leur concentration semble déterminante pour le milieu aquatique. Malheureusement, il n'existe pas encore de méthode simple pour obtenir cette variable. Les valeurs indiquées pour le Co sont basées sur les analyses voltamétriques et des calculs thermodynamiques complexes (Qian et al., 1998). La difficulté est liée à la complexité des substances organiques du système aquatique, qui proviennent concurremment des rivières même et des sols érodés des bassins versants (Buffle, 1988). Le comportement du Co dans une rivière avec une concentration de matière organique forte est donc nettement différent par rapport à une rivière à faible concentration organique. Ceci peut expliquer une partie des différences observées pendant 4 décennies d'études sur le comportement du ${ }^{60} \mathrm{Co}$ dans les différents sites du monde (p.e. Aston et Duursma, 1973; Baudin et Nucho, 1992; Benes et al., 1889; Cerling et Turner, 1982; Cundy et Croudace, 1995; Foulquier et al., 1989; Hesslein, 1987; Lambrechts et Foulquier, 1985; Mahara et Kudo,

Tableau 6. - Répartition du Co entre phase libre et association avec des ligands organiques $(\mathrm{CoL})$ et des particules $\left(\equiv \mathrm{FeOCo}{ }^{+}\right.$) modélisée pour un $\mathrm{pH}=8$ et deux concentrations de ligands $(L)$ différentes.

Table 6. - Partitioning of $\mathrm{Co}$ between the free, dissolved phase and association with organic ligands $(\mathrm{CoL})$ and particles $\left(\equiv \mathrm{FeOCO}^{+}\right)$modeled at $\mathrm{pH} 8$ for two different ligand concentrations (L).

(L) $[\mathrm{nM}]$

1.4

7.6
$\mathrm{Co}^{2+}[\%]$

3.4

0.5
(CoL) [\%]

49.5

93.2
(=FeOCo+) [\%]

47

6.3 


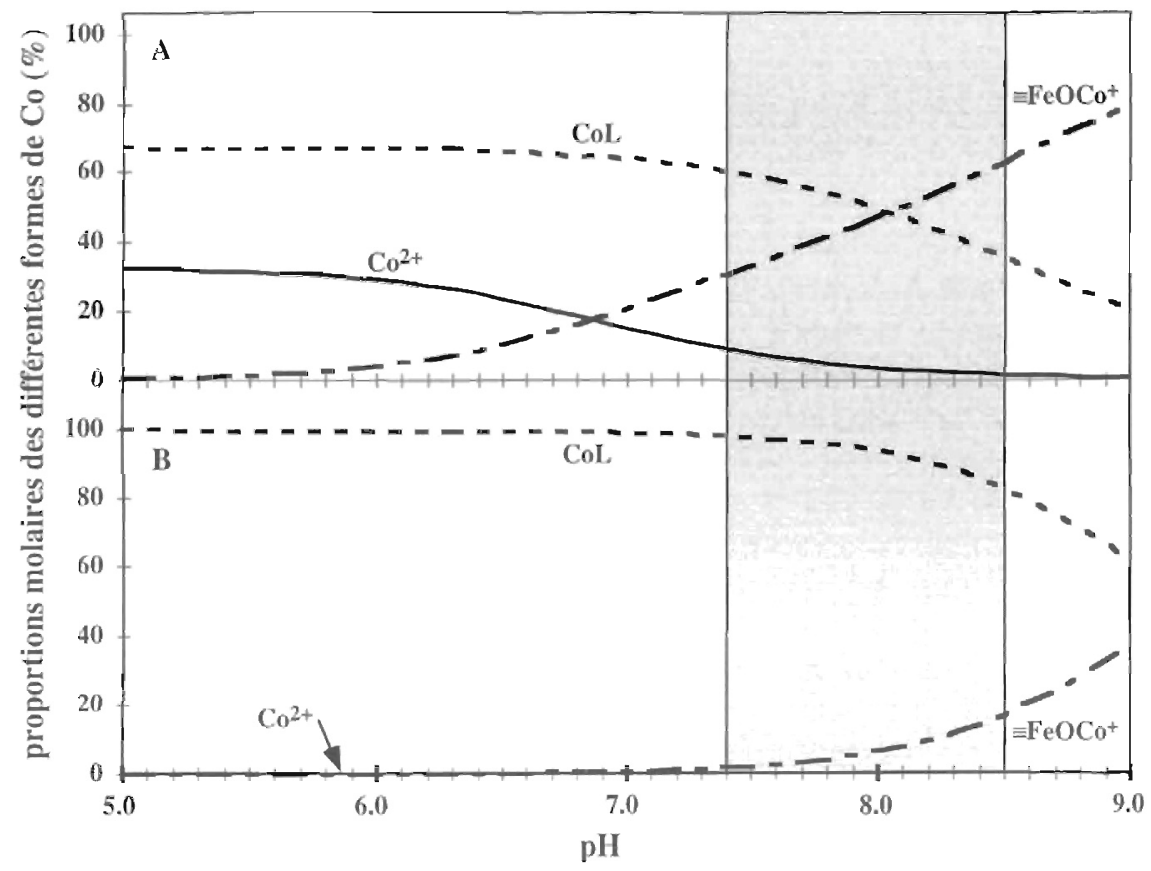

Fig. 8. - Modèle de la spéciation du Co dans un système de compétition entre adsorption et complexation organique. Les résultats obtenus pour les deux cas sont basés sur les données résumées dans le Tableau 5, à part la concentration des ligands qui est dans le cas $\mathrm{A}$ égale à $1.4 \mathrm{nM}$ et dans le cas $\mathrm{B}$ égale à $7.6 \mathrm{nM}$.

Fig. 8. - Speciation modeling of $\mathrm{Co}$ in a system with competition between adsorption and organic complexation. Results obtained for both cases are based on the parameters summarized in Table 5. with the exception of the ligand concentration, which is in case A equal to $1.4 \mathrm{nM}$ and in case $\mathrm{B}$ equal to $7.6 \mathrm{nM}$.

1980; Mundschenk, 1992; Nucho et al., 1988; Pally et al., 1986; Vertacnik et al., 1979).

\subsection{Intérêt pour la surveillance des installations nucléaires}

Dans une publication récente Mundschenk (1996a) a conclu que dans un système aquatique à l'équilibre la majorité des radionucléides (Co inclus) est adsorbée aux particules. Cette conclusion avait des con- séquences pour la mise au point d'un système de surveillance des installations nucléaires, par exemple une préférence pour les analyses des sédiments. Le faible pourcentage de ${ }^{60} \mathrm{Co}$ adsorbé aux particules en aval de la centrale de Mühleberg démontre que les sédiments seuls ne peuvent pas servir d'outil de contrôle et que la phase dissoute doit aussi être prise en considération. En Suisse, nous avons terminé l'installation de trois 
systèmes d'échantillonnage qui collectent entre 100 et 200 I d'eau brute par mois selon le débit du fleuve. L'échantillon est ensuite traité par précipitation d'hydroxyde de fer et floculation de montmorillonite. L'activité mesurée en $\mathrm{Bq} / \mathrm{m}^{3}$ est ensuite multipliée par le débit mensuel de la rivière pour être comparée aux déclarations des centrales nucléaires. Un lieu d'échantillonnage se trouve en aval de le centrale de Mühleberg près de Hagneck (Fig. 1; Albrecht et Weidmann, 1998; Albrecht 1989).

\subsection{Implications radioécologiques}

Les études d'impact des centrales nucléaires portaient essentiellement sur l'eau de la rivière, les sédiments, les poissons et les végétaux aquatiques (p.e. Lambrechts et al., 1992). La mobilité du cobalt implique la possibilité de sa présence dans les nappes aquatiques en contact avec l'eau de rivière, surtout en cas d'infiltration, soit naturelle ou artificielle. Deux cas précis sont en cours d'évaluation. Dans un premier cas, en aval de la centrale de Mühleberg où l'eau de l'Aar s'infiltre régulièrement dans la nappe aquatique, des traces de ${ }^{60} \mathrm{Co}$ ont été mesurées dans l'eau souterraine $\left(6 \pm 0.8 \mathrm{~Bq} \mathrm{~m}^{-3}\right)$. Un deuxieme cas traite une installation d'enrichissement d'eau souterraine pres de Bâle, ou l'eau du Rhin peut s'infiltrer dans la nappe. Dans un échantillon d'eau potable une activité de $0.5 \pm 0.1 \mathrm{~Bq} \mathrm{~m}^{-3}$ a été mesurée (Albrecht, 1998). Ces activités sont largement inférieures à la valeur de tolérance suisse de $10000 \mathrm{~Bq} \mathrm{~m}$, mais elles démontrent clairement la mobilité des complexes organiques de Co et la nécessité d'inclure les eaux des nappes souterraines dans une étude d'impact lorsqu'une infiltration est supposée.

\subsection{Applicabilité pour autres radionucléides}

La méthode présentée dans ce travail est également applicable pour autres radionucléides. Les radionucléides des métaux de transition, comme ${ }^{54} \mathrm{Mn}$ ou ${ }^{65} \mathrm{Zn}$ et des métaux alcalins, comme ${ }^{137} \mathrm{Cs}$ ou ${ }^{134} \mathrm{Cs}$ existe dans les effluents des centrales et ont aussi un intérêt radioécologique. Les résultats sur leur comportement dans les systemes aquatiques en Suisse sont moins nombreux dus à leurs faibles concentrations. Ces métaux participent, comme le fait le Co dans les réactions d'adsorption et de complexation organique. Pour la modélisation il est nécessaire de consulter la littérature afin de trouver des renseignements sur les concentrations respectives et les constantes d'équilibres. Pour le radiocaesium la démarche est plus complexe, car Cs peut exister dans un systeme aquatique sous forme d'ion (surtout à courte distance en aval du lieu de décharge) ou fortement adsorbé aux particules de sol, érodé dans le bassin versant. Surtout les méthodes d'enrichissement chimiques et de séparations par résines doivent être adaptées aux radionucléides en question. 


\section{REMERCIEMENTS}

Je remercie le personnel de la centrale nucléaire de Mühleberg et des centrales hydroélectriques de la 'Kraftwerkgruppe Seeland' pour leur support durant les années de la recherche et surtout durant les périodes des investigations sur le terrain. L' application des modèles aquatiques et chimiques a été possible grâce à l'inspiration et l'aide de P. Reichert, de G. Goudsmit et de L. Sigg. Merci à toutes celles et tous ceux qui ont participé aux travaux de terrain et de laboratoire, particulièrement à $A$. Lück et $Y$. Weidmann. Ce travail est financé par la Division principale de la Sécurité des Installations Nucléaires (HSK). Merci à C. Murith et le comité éditorial pour ces commentaires, qui ont aidé à améliorer le texte et à $E$. Steffen pour avoir révisé le texte français.

\section{RÉFERENCES \\ BIBLIOGRAPHIQUES}

Albrecht A., Beer J., Gloor M., Fischer A. et Mattle N., 1994. Aquatic transport behavior of cobalt deduced from nuclear reactor derived ${ }^{60} \mathrm{Co}$. Mineralogical magazine. 58A : 13.

Albrecht A., Reichert P., Beer J. et Lück A., 1995. Evaluation of the importance of reservoir sediments as sinks for reactor-derived radionuclides in riverine systems. Journal of Environmental Radioactivity. 28 : 239-269.

Albrecht A., Weidmann Y. Lück A., Kobler D. et Beer J., 1996. Radioaktivitätsüberwachung im aquatischen Milieu;
Anpassung der Beprobungsstrategie an neue Kenntnisse. In: Völkle, $H$. (ed.), Umweltradioaktivität und Strahlendosen in der Schweiz 1995. Bern, Bundesamt für Gesundheitswesen, SUER, Chemin du Musée, $\mathrm{CH}-1700$ Fribourg. pp. B.3.9.1-11.

Albrecht A., Goudsmit G., Qian J., Sigg L., Xue $H$., Kobler D., Lück $A$. et Weidmann Y., 1997. Transport de cobalt dans le lac de Bienne; A la recherche de l'Aar. EAWAG News. 43: 21-24.

Albrecht A., Reiser R., Lück A., Stoll J.M. and Giger W., 1998. Radiocesium dating of sediments from lakes and reservoirs of different hydrological regimes. Environ. Sci. Techn. 32 : 1882-1887.

Albrecht A., 1998. The behavior of nuclear reactor derived metallic radionuclides in the aquatic system of Switzerland. Swiss Federal Institute for Environmental Science and Technology (EAWAG), $\mathrm{CH}-8600$ Dübendorf, Schriftenreihe der EAWAG \#13: pp. 120.

Albrecht A. and Weidmann Y., 1998. Umgebungsüberwachung im Spannungsfeld von Reglement und Anpassung am wissenschaftlichen Kenntnisstand. in : M. Winter, K. Henrichs and $\mathrm{H}$. Doerfel (eds.), Radioaktivität in Mensch und Umwelt. Lindau, TUV-Verlag. II : 802-807.

Albrecht A., Goudsmit G. et Zeh M., 1999. Importance of lacustrine physical factors on the distribution of anthropogenic ${ }^{60} \mathrm{Co}$ in Lake Biel. Limnol. Oceanogr. 44/1 : 196-206.

Albrecht A., 1999. Cobalt : abundance and geochemistry. In : Marshall, C.P. (ed.), Encyclopedia of geochemistry. Chapman \& Hall. in press.

Amiard-Triquet C. et Saas A., 1979. Modalités de la contamination de deux chaines trophiques dulçaquicoles par le Cobalt 60. II. Contamination simultanée des organismes par l'eau et la 
nourriture. Water, Air and Soil Pollution. 12 : 141-153.

Aston S.R. et Duursma E.K., 1973. Concentration effects on ${ }^{137} \mathrm{Cs},{ }^{55} \mathrm{Zn}$, ${ }^{50} \mathrm{Co}$ and ${ }^{1356} \mathrm{Ru}$ sorption by marine sediments, with geochemical implications. Netherlands Journal of Sea Research. $6: 225-240$.

Ballestra S., Pettersson H., Gastaud J., Parsi P. et Vas D., 1997, Intercomparison of radionuclide measurements on marine sediment IAEA-315, IAEAMEL, B.P. 800, 98012 Monaco Cedex, December 1997,

Baudin J.P. et Nucho R., 1992. ${ }^{50} \mathrm{Co}$ accumulation from sediment and planktonic algae by midge larvae (Chironomus luridus). Environmental Pollution. 76 : 133-140.

Benes P., Jurak M. et Kuncova M., 1889. Factors affecting interaction of radiocobalt with river sediments. Journal of Radioanalytical et Nuclear Chemistry, Articles. 132 : 209-223.

Buesseler K.O., Bauer J.E., Chen R.F., Eglinton T.I., Gustafsson O., Landing W., Mopper K., Moran S.B., Santschi P.H., VernonClark R. et Wells M.L., 1996. Sampling marine colloids using Cross-Flow Filtration: Overview and results from an intercomparison study. Marine Chemistry. 55 (1-2): 1-32.

Buffle J., 1988. Complexation reactions in aquatic systems: An analytical approach. New York, Ellis-Horwood. pp. 692.

Buffle J., Perret D. et Newman M., 1992. The use of filtration and ultrafiltration for size fractionation of aquatic particles, colloids, and macromolecules. In: J. Buffle et H.P. van Leeuwen, (ed.), Environmental particles. Boca Raton. Lewis Publishers. pp. 171-230.

Cerling T.E. et Turner R.R., 1982. Formation of freshwater $\mathrm{Fe}-\mathrm{Mn}$ coatings on gravel and the behavior of "Fo, "Co $\mathrm{Cr}$. and ${ }^{13 /} \mathrm{Cs}$ in a small watershed. Geochimica et Cosmochimica Acta. 46 : 1333.
Cundy A.B. et Croudace I.W., 1995. Physical and chemical association of radionuclides and trace metals in estuarine sediments: an example from Poole Harbour, Soutern England. J. Environ. Radioactivity. 29 : 191-211.

Dzombak D.A. et Morel F.M.M., 1990. Surface complexation modelling. Hydrous ferric oxide. New York, Wiley-Interscience. pp. 331.

Ferrer M.C., 1983. Étude expérimentale du comportement de huit radionucléides artificiels dans le cours terminal du Rhône, Thèse, Université de Bordeaux 1: pp. 147.

Foulquier L., Baudin J.P. et Lambrechts A., 1989. Données sur les transfert du ${ }^{137} \mathrm{Cs}$ et $d \mathrm{du}^{50} \mathrm{Co}$ dans un écosystème fluvial: le Rhône. revue des SCIENCES DE L'EAU. 2 : 641-658.

Garnier J.-M., Pham M.K., Ciffroy P. et Martin J.-M., 1997. Kinetics of trace metal comlexation with suspended matter and filtrable ligands in fresh water. Env. Sci. Techn. 31 : 1597-1606.

Hesslein R.H., 1987. Whole lake metal radiotracer movement in fertilized lake basins. Can. J. Fish. Aquat. Sci. 44 : 74-82.

Hurd D.C. et Spencer D.W., 1991, Marine particles: Analysis and Characterization, Washington,

Lambrechts A. et Foulquier L., 1985. Experimental study of the transfers of a radionuclide mixture between water, sediment and fish. In : T.H. Sibley et C. Myttenaere, (ed.), Application of distribution coefficients to radiological assessment models. London \& New York, Elsevier. pp. 336-350.

Lambrechts A., Foulquier L. et Pally M., 1992, Plan Rhône; Synthèse des connaissances sur la radioécologie du Rhône, Commissariat à l'Energie Atomique, Institut de Protection et de Sureté Nucléaire. Cadarche.

Leonard K.S., McCubbin D. et Harvey B.R., 1993a. Chemical speciation and environmental behaviour of ${ }^{50} \mathrm{Co}$ 
discharged from nuclear establishment. J. Environ. Radioactivity. 20 : 1-21.

Leonard K.S., McCubbin D. et Harvey B.R., 1993b. A radiochemical procedure for the determination and speciation of radiocobalt in environmental waters. The science of the total environment. 130/131: 237-251.

Li Y.-H., Burkhardt L., Buchholtz M., O'Hara P. et Santschi P.H., 1984. Partition of radiotracers between suspended particles and seawater. Geochimical et Cosmochimica Acta. 48 : 2011-2019.

Mahara Y. et Kudo A., 1980, Mobility and Retention of ${ }^{60} \mathrm{Co}$ in Soils in Coastal Areas, 5th International Congress IRPA, 319-322

Marschner H., 1995. Mineral nutrition of higher plants. London, Academic Press. pp. 889.

Martell A.E. et Smith R.M., 1989. Critical stability constants. New York, Plenum Press.

Miller M.C., McCave I.N. et Komar P.D., 1977. Threshold of sediment motion under unidirectional currents. Sedimentology. 24 : 507-527.

Moffett J.W. et Ho J., 1996. Oxidation of cobalt and manganese in seawater via a common microbially catalyzed pathway. Geochim. Cosmochim. Acta. $60: 3415-3424$.

Morel F.M.M. and Hering J.G., 1993. Principles and applications of aquatic chemistry. New York, John Wiley \& Sons. pp. 588.

Müller B., 1996, ChemEQL, a program to calculate chemical speciation equilibria, titrations, dissolution, precipitation, adsorption, simple kinetics and pX-pY diagrams, EAWAG, Feb. 1996, manual.

Mundschenk H., 1992. Study of the longrange effects of radioactive effluents from nuclear power plants in the Rhine River using ${ }^{58} \mathrm{Co}$ and ${ }^{60} \mathrm{Co}$ as tracers. J. Environ. Radioactivity. 15 : 51-68.

Mundschenk H., 1996a. Occurrence and behaviour of radionuclides in the Moselle River-Part II: Distribution of radionuclides between aqueous phase and suspended matter. J. Environ. Radioactivity. $30: 215-232$.

Mundschenk H., 1996b, Vergleichsanalyse Radionuklide im Sediment 1995, Bundesanstalt für Gewässerkunde D 56068 Koblenz Kaiserin-Augusta-Anlagen 15-17, Januar 1996.

Nucho R., Rambaud A., Foulquier L. et Baudin J.-P., 1988. Bioaccumulation du ${ }^{50} \mathrm{Co}$ par une algue planctonique Scenedesmus obliquus Türps (Kütz). Influence du stade de développement de la culture sur la fixation du radionucléide. Acta Oecologica/Oecologia Applicata. $9:$ 111-125.

Pally M., Baudin J.P., Fritsch A.F., Lambrechts A., Maurel-Kermarrec A. et Foulquier L., 1986. Étude des formes chimiques du cobalt-60 au cours de diverses expérience de transfert entre l'eau et des organismes aquatique. Sciences de l'eau. 5 : 273-290.

Qian J., Xue H., Sigg L. et Albrecht A., 1998. Complexation of cobalt by natural ligands in freshwater. Env. Sci. Technol. 32 : 2043-2050.

Reichert P., 1994a. AQUASIM - A tool for simulation and data analysis of aquatic systems. Water Sci. Tech. $30: 21$ 30.

Reichert P., 1994b, Concepts underlying a computer program for the identification and simulation of aquatic systems, Swiss Federal Institute for Environmental Science and Technology (EAWAG), $\mathrm{CH}-8600$ Dübendorf, Schriftenreihe der EAWAG

Sigg L., W. Stumm et P. Behra, 1994. Chimie des milieux aquatiques. Paris, Masson. pp. 391.

Smith R.M. et Martell A.E., 1974. Critical stability constants. New York, Plenum Press. vols. 1-4. 
Stumm W. et Morgan J.J., 1996. Aquatic Chemistry; Chemical equilibria and rates in natural waters. New York, John Wiley \& Sons. pp. 1022.

Tipping E. et Hurley M.A., 1992. A unifying model of cation binding by humic substances. Geochimica et Cosmochimica Acta. 56 : 3627-3641.

Vertacnik A., Strohal P. et Lulic L., 1979. Fixation of ${ }^{60} \mathrm{Co}$ et ${ }^{137} \mathrm{Cs}$ on natural sorbents from pheol - polluted water. Health Physics. 36 : 491-496.
Wedepohl K.H., 1969, 1972, 1974, 1978, Handbook of Geochemistry, Berlin, Heidelberg. New York, 27-A-1 H.

Westall J., Zachary J.L. et Morel F., 1976, MINEQL, a computer program for the calculation of chemical equilibrium composition of aqueous systems, Ralph M. Parsons Lab., MIT, 1976, Technical note

Westall J.C., 1986, MICROQL, A chemical equilibrium program in basic, Oregon State University. 